\title{
Abstracts from the 19th International Congress on Twin Studies, 11-14 November 2021
}

\section{Exceptional Sibships and Curious Couples: General Intelligence Findings From Chinese Twins Reared Apart and Together and Virtual Twins}

Nancy L. Segal

California State University, Fullerton, CA, USA

Introduction: Reared-apart twin research and adoption data have supported genetic influence on general intelligence. However, reared-apart twin studies have compared the test scores of reunited adult twins only. The present study examined genetic and environmental influences on general intelligence via three novel sibships who vary in genetic and environmental relatedness. Method: The study sample included young Chinese twins reared apart (CTA, $n$ [pairs] =23), Chinese twins reared together (CTT, $n$ [pairs] $=50)$ and Virtual twins (VT, i.e., same-age unrelated siblings reared together from infancy, $n$ [pairs] $=169$ ). This study is the first to prospectively track the development of young separated twins. Hypotheses were that (1) CTAs would show reduced intellectual resemblance relative to CTTs, given the CTAs' separate rearing, and that (2) both twin groups would show greater intellectual resemblance than the VTs, due to the twins' genetic relatedness. Participants completed the age-appropriate version of the Wechsler Intelligence Scales, administered to pair members by separate examiners. Results: (1) CTT twins showed greater IQ resemblance than CTA twins, reflecting shared environmental effects; and (2) both twin groups showed greater resemblance than VTs, demonstrating genetic influence; only the CTT-VT comparison reached statistical significance $(p<.001)$. Interestingly, VT pairs composed of two adoptees were significantly more alike in intelligence than VT pairs composed of one biological child and one adoptee $(p<.001)$. Conclusions: Genetic and environmental effects on general intelligence were demonstrated. Parents and educators should anticipate behavioral similarities in closely related children, and developmental variations in unrelated siblings.

\section{A Transcriptomic-Wide Association Study of Spontaneous Dizygotic Twinning and its Relation to the Fertility Trait Number of Children Ever Born}

\footnotetext{
Nikki Hubers ${ }^{1}$, Eske Derks ${ }^{2}$, Zac Gerring ${ }^{2}$ and Dorret I. Boomsma ${ }^{1}$

${ }^{1}$ Department of Biological Psychology, Vrije Universiteit, Amsterdam, the Netherlands and and ${ }^{2}$ QIMR Berghofer Medical Research Institute, Brisbane, Qld, Australia
}

Introduction: Spontaneous dizygotic (DZ) twinning is defined as conceiving a DZ twin without the help of artificial reproductive techniques. DZ twinning occurs when two zygotes are simultaneously conceived and developed during one pregnancy and is relatively common, with 13 per 1000 births on average. This study aimed to gain insight into the genetic disposition of $\mathrm{DZ}$ twinning and into the genetic overlap between DZ twinning and fertility. More insight is needed since $\mathrm{DZ}$ twinning has been suggested to be genetically associated with both fertility and infertility. Methods: A Transcriptomic-wide association study (TWAS) was performed using the latest DZ twinning GWAMA summary statistics. MetaXcan was employed to perform the TWAS applying Joint-Tissue Imputation (JTI). Subsequently, the TWAS was replicated in the fertility trait Number of Children ever Born (NEB) and the genetic correlation between DZ twinning and NEB was calculated using LD Score Regression. Results: The TWAS linked multiple new genes such as PCBP2, GOLGA8T and long noncoding RNA fragments to DZ Twinning. Additionally, the TWAS replicated several previously associated genes with known reproductive function including ARL14EP, FSHB and SMAD3. Several identified genes were previously linked to infertility traits such as Polycystic Ovary Syndrome and recurrent pregnancy loss. Furthermore, no genetic overlap in the TWASs results and no significant genetic correlation $(r=.089, S E=0.063, p=.155)$ was found between DZ twinning and NEB. Conclusion: Overall, the study sheds more light on the genetic architecture of DZ twinning and emphasises the complicated relationship between DZ twinning and fertility.

\section{The Evolutionary Biology of Human Twinning}

Scott Forbes

University of Winnipeg, Winnipeg, MB, Canada

The modal brood size of humans is one, but twins, triplets and even higher order multiples fall within the normal range of variation in brood size. Bearing twins has the obvious advantage of increasing the rate of reproductive output but at what cost? The burden of caring for twins, both pre- and postnatal, increases over singletons; twinning squeezes (literally) space within the womb; it increases the demand for micro- and macronutrients. Without access to adequate resources, there is the potential for a quantity-quality trade-off that is common to other organisms. Twinning in humans has both genetic and environmental causes. Dizygotic twinning especially is a complex multifactorial trait that is in part heritable, but also affected by maternal phenotype. Taller, heavier women are more prone to twinning; and dizygotic twinning is more common in older women, where it appears to serve as an insurance strategy for a rising incidence of aneuploidy. Behavioral ecologists would describe dizygotic twinning in humans as a conditional reproductive strategy: those women best able to bear the additional burden of twins are 
those most prone to twinning, what might be described as a maternal evolutionary 'choice'. The incidence of twinning across the globe has increased dramatically with the advent of assisted reproduction (AR) and some AR techniques, such as in vitro fertilization and the transfer of multiple embryos, are designed to increase the take-home baby rate by bypassing built-in mechanisms of maternal quality control. Twins are effectively imposed upon mothers, rather than physiologically 'chosen'.

\section{University of Sao Paulo Twin Panel}

Emma Otta

Instituto de Psicologia University of São Paulo. São Paulo, Brazil

The Painel USP de Gêmeos (University of Sao Paulo Twin Panel), based at Instituto de Psicologia, Universidade de São Paulo, started formally in 2017, and has currently 6479 individuals registered (97.5\% twins and $2.5 \%$ higher-order multiples). It comprises a volunteer sample of registered individuals (1691 adults and 4788 children and adolescents). Our main aim is to conduct twin research on psychological processes and behavior. We translated and validated Zygosity Questionnaires and Sibling Relationship Questionnaires. Currently studies are underway to investigate voice similarity in twins, facial expression signatures, and collaborative problem solving. In partnership with Professor Nancy Segal we started studying twins raised apart and reunited in adulthood. A partnership was established with colleagues from the USP School of Dentistry and the USP Medical School. A study on CARies DEtection and management in Children (CARDEC) is conducted by a research team headed by Professor Fausto Medeiros Mendes. We will start studying the reaction of twin children (7-12 years old) to dental treatment, through self-report of environmental sensitivity, and of dental anxiety, and physiological and behavioral measures. The Psychological Care Branch of Painel USP de Gêmeos directed by Professor Maria Livia Moretto offers free counseling and psychological services to people referred by our registry. The clinical setting is also fostering research. The University of São Paulo is the largest higher education and research institution in South America, and the Painel USP de Gêmeos has great potential for fostering research on twin-related issues from a psychological perspective in Brazil and South America.

\section{Monozygotic Twins: Developmental Dilemmas}

Joan A. Friedman

Institute of Contemporary Psychoanalysis, Los Angeles, CA, USA

Growing up as a monozygotic twin comes with special privileges as well as distinct developmental challenges. I will discuss three psychological variables that may impact the emotional wellbeing of twin connections as they mature: caretaking, co-dependency, and competition. The manner in which these issues are addressed will influence attachment patterns outside of the twinship.

\section{The Twinlife Study: Monochorionic Twins as a Natural Experiment to Unravel The Epigenetic Mechanisms Underlying the Developmental Origins of Disease}

Bastiaan T. Heijmans ${ }^{1}$, Pia Todtenhaupt ${ }^{1,2}$, Sophie G. Groene ${ }^{1,2}$, Christiaan de Bruijn ${ }^{2}$, Erik W. van Zwet ${ }^{1}$, Monique C. Haak ${ }^{3}$, Arno A. W. Roest ${ }^{2}$, Jeanine M. M. van Klink ${ }^{2}$, Melissa van $\mathrm{Pel}^{4}$ and Enrico Lopriore ${ }^{2}$

${ }^{1}$ Department of Biomedical Data Sciences, Leiden University Medical Center, the Netherlands, ${ }^{2}$ Department of Pediatrics, Leiden University Medical Center, the Netherlands, ${ }^{3}$ Department of Obstetrics, Leiden University Medical Center, the Netherlands and ${ }^{4} \mathrm{NecstGen}$, Leiden, the Netherlands

Lifelong health is thought to be partially set during intrauterine life by persistent epigenetic changes induced by the prenatal environment. To evaluate this hypothesis, we initiated a prospective longitudinal study in monochorionic (MC) twins: The Twinlife study. MC twins are monozygotic, thus in origin genetically identical, and share a single placenta. Although MC twins have many environmental factors in common, in one-third of the MC twin pairs, one fetus has significantly less access to nutrients and resources during pregnancy than its co-twin often resulting in a significant discordance in prenatal growth. Hence, MC twins constitute a unique natural experiment to study the influence of the prenatal environment on health. In Twinlife, we will chart intrapair differences in DNA methylation and transcription focusing on mesenchymal stromal cells (MSC) isolated from umbilical cord (UC) as an advanced proxy of epigenetic dysregulation relevant for long-term health consequences. For this purpose, we established a biobank that currently containing MSCs from $>80 \mathrm{MC}$ twin pairs and is still expanding. To allow a comparison of UC-derived MSCs within and between twin pairs standardization of isolation, expansion and characterization protocols is critical. By comparing various isolation and expansion methods, we developed a protocol optimized for UC-dissection method, culture medium use, cell expansion and immunophenotyping, resulting in a standardized and highly reproducible procedure. Currently, we are assessing the differentiation capacity of the UC-MSCs into different end cells including adipocytes, osteoblasts, chondrocytes and neuronal cells. Through epigenetic, gene expression and functional studies on genetically identical MSCs, we aim to gain more insight into the mechanism underlying the long-term health impact of an adverse intrauterine environment.

\section{Copy Effect Hypermetropic Anisometropia in a Pair of Monozygotic Twins: A Case Report}

Fatma Sümer and Mehmet Gökhan Aslan

Recep Tayyip Erdogan University, Rize, Turkey

Introduction: The aim of this case report is to report hyperopic anisometropia with copy-image in monozygotic twins. Methods: In March 2021, a 5-year-old identical twin was seen, who was referred to Recep Tayyip Erdogan University Training and Research Hospital Ophthalmology Department with the diagnosis of amblyopia in their left eyes from an external center. Both twins had a full ophthalmic examination, which included visual acuity testing, ocular motility testing, cycloplegic refraction, and fundus examination. Results: On examination, 'copy image' hypermetropic anisometropia was discovered. Twin 1 had anisometropia with hypermetropic astigmatism in the left eye. His cycloplegic refraction was $+1.00(-0.75 \times 75)$ in the right eye and $+6.50(-1.00 \times 175)$ in the left eye. Similarly, twin 2 had anisometropia with hypermetropic astigmatism in the left eye. His cycloplegic refraction was $+6.75(-1.00 \times 180)$ in the left eye and $+1.25(-0.50 \times 90)$ in the right eye. The best-corrected visual acuity was $20 / 60$ in the amblyopic eyes and $20 / 20$ in the unaffected eyes. There was no ocular deviation. In either patient, a slit-lamp microscopic examination revealed no abnormalities in the anterior parts of either eye. Fundoscopic examination revealed no abnormalities. No abnormal ocular movements were demonstrated. Conclusion: As far as we have reviewed in the literature, previous studies with twins were mostly concerned with mirror effect myopic anisometropia and hyperopic anisometropia, whereas ipsilateral amblyopia and 
anisometropia were not reported in monozygotic twins. This case underscores the possible genetic basis of hypermetropic anisometropia.

\section{Prevalence of Twinning: Mother's Age and Fertility}

András Pári'1,2

${ }^{1}$ Mária Kopp Institute for Demography and Families (KINCS), Budapest, Hungary and ${ }^{2}$ University of Pécs, Doctoral School of Sociology and Demography, Pécs, Hungary

Introduction: The number of multiple births vary from region to region. In general, we know that the combined effect of biological or natural factors and/or artificial treatments also increase the chances of multiple conceptions. The frequency of the number of multiple births reached the level of 28 in 1000 births in 2018 worldwide. Methods: The number of multiple births is collected from UN database of live births by plurality. Twin birth rates (TBR) have been estimated because no data are available in some countries. TBRs were standardized on World Bank 2018 data such as total fertility rate (TFR) and average age of mother (AAoM) at childbirth (UN 2008 country-specific data) and infant mortality rate (2015-2020 UN average). Results: An estimated average of 3.9 million children of multiple birth were born (yearly) between 2015 and 2020, bringing the average TBR to $27.93 \%$. The AAoM of 30 years and older shows an average of $2.86 \mathrm{TFR}$, which is 0.41 higher than the world's average $\mathrm{TFR}=2.45$. The TFR is 1.83 at $\mathrm{AAoM}$ of 27 years and younger. TBR is higher in countries where the ratio of 'older' $(30+)$ mothers is higher (TBR $=32.37 \%$ ) and lower in countries where the proportion of mothers aged 27 and 'younger' is lower (TBR $=20.17 \%$ o). Conclusions: The fertility and the number of previous births have a statistically significant effect on TBR. There is a strong correlation between TBR and TFR $\left(R^{2}=.7\right)$ and some correlation $\left(R^{2}=.14\right)$ between TBR and AAoM worldwide.

\section{Treating Twins as a Couple in Parental Communication}

Rita Hegedüs ${ }^{1,2}$ and Zsófia Drjenovszky ${ }^{2}$

${ }^{1}$ Corvinus University of Budapest, Budapest, Hungary and ${ }^{2}$ Károli Gáspár University of the Reformed Church in Hungary, Budapest, Hungary

Introduction: Much is known about the development of twins' speech and the background factors; among them, the treatment of children as pairs stands out. We look at how the social expectation that twin children are valued only as a couple is reflected in parents' communication practices. Method: We conducted an online survey of twins' parents in 2019. We collected data from 135 pairs, through a questionnaire with both closed and open questions. We use a mixed methodology, employing quantitative and qualitative content analysis and cross-tables. Results: We examine the prevalence of paired names, the lack of uniqueness of the names given to children, and the individual communication with the child. In our sample, the prevalence of paired names is low in terms of parental motivation (18 cases), but our own coding suggests that the practice is more present (45 cases). Frequent joint addressing of children is common among about one-fifth of parents (31 cases), although sometimes it can be applied to non-twin siblings. Parents generally know their children require special attention and many of them try to address them individually. The more educated parents are more self-critical, but when asked about practice, the difference disappears and sometimes the relationship reverses direction. Conclusions: The pair isolation of twins is affected by the naming and addressing used by parents. To clarify the relationship between lack of individual treatment and stunting in speech development, we see a need for further qualitative case study and a larger sample of targeted questionnaires.

\section{Impaired Motor Function in Children and Adolescents with CHD in a Direct Twin Comparison - But Young Children With CHD Better Than Adolescents}

Julia Remmele ${ }^{1,3}$, Paul C. Helm ${ }^{2}$, Fritz R. Oberhoffer ${ }^{3}$, Ulrike M. M. Bauer ${ }^{2}$ and Peter Ewert ${ }^{1,4}$

${ }^{1}$ German Heart Center Munich, Technical University of Munich, Department of Congenital Heart Disease and Pediatric Cardiology, Munich, Germany, ${ }^{2} \mathrm{DZHK}$ (German Center for Cardiovascular Research), National Register for Congenital Heart Defects, Berlin, Germany, ${ }^{3}$ Technical University of Munich, Chair of Preventive Pediatrics, Munich, Germany and ${ }^{4} \mathrm{DZHK}$ (German Centre for Cardiovascular Research), Partner Site Munich Heart Alliance, Munich, Germany

Introduction: Newborns and infants with congenital heart defect (CHD) often show delayed neurodevelopment. It seems obvious that the operations and treatments of the heart defect, especially on motor skills, are responsible for this delay. It is unclear how the children will develop in the future and how they would have developed without the CHD. Therefore, we compared motor function of twin siblings with and without CHD. Methods: Motor function was measured as part of the nationwide ongoing twin study 'SameSame', investigating neurodevelopment. 44 pairs of twins (one with CHD the other without, 50 girls (56.8\%), $8.94 \pm 4.3$ years) have completed the Movement Assessment Battery for Children-II so far. The different age groups (A: 3-6 years, $n=32$; B: 7-10 years, $n=28$; C: $11-16$ years, $n=28$ ) consist of eight age-specific tasks covering the areas of manual dexterity, ball skills, static and dynamic balance and a total motor score. Results: Children with CHD showed reduced scores in manual dexterity $(p=.014)$, in static and dynamic balance $(p=.001)$ and totalmotor-score $(p=.004)$ in the direct comparison with their healthy twin. Group A showed a significant difference in ball skills $(p=.024)$, group $\mathrm{B}$ in manual dexterity $(p=.032)$ and group $\mathrm{C}$ in manual dexterity $(p=.030)$, ball skills $(p=.026)$, static and dynamic balance $(p=.047)$ and total-motor-score $(p=.002)$ in the burden on children with CHD. Conclusions: Children and especially adolescents with CHD show deficits in motor function compared to their healthy twin siblings. Surprisingly adolescents show clear deficits in all motor function areas. This demonstrates that patients with CHD need lifelong support and assistance to avoid or compensate for such performance deficits.

\section{The Efficacy of Cell-Free Fetal DNA-Based Noninvasive Prenatal Testing In Twin Pregnancies}

Maria Butenko, Olesya Bespalova, Pachulia, and Glotov

FSBSI, The Research Institute of Obstetrics, Gynecology and Reproductology named after D. O. Ott of the Ministry of Healthcare of the Russian Federation, Saint Petersburg, Russia

Introduction: Cell-free fetal DNA (cffDNA) non-invasive prenatal testing (NIPT) is rapidly expanding. NIPT shows high sensitivity and specificity in singleton pregnancy, but there is little evidence of the efficacy of NIPT for the twin pregnancy. The objective of this study was to determine efficacy of cffDNA-based NIPT for trisomies 21, 18 and 13 in twin pregnancies. Materials and Methods: A search of literature has been carried out in Medline, Web of Science, Embase and Cochrane Library databases. After reviewing the full article, 19 studies were eligible. Quality assessments were conducted with the 
Quality Assessment Tool for Diagnostic Accuracy Studies-2 checklist. Data analysis were performed by meta-analysis using Review Manager 5.4.1 (Cochrane col., UK). Results: Nineteen eligible studies were enrolled in this meta-analysis. There were 132 cases of trisomy 21, 40 cases of trisomy 18, 12 cases of trisomy 13 and 6954 cases of euploids in total. For trisomy 21, NIPT showed the sensitivity, specificity, positive likelihood ratio, negative likelihood ratio were 0.98 , $0.99,1116,0.017$, respectively. For trisomy 18 , the sensitivity, specificity, positive likelihood ratio, negative likelihood ratio were 0.97, $0.99,915,0.029$, respectively. For trisomy 13 , the sensitivity, specificity, positive likelihood ratio, negative likelihood ratio were 0.875 , $0.99,929,0.125$, respectively. Conclusions: The efficacy of NIPT for trisomy 21 and 18 in twin pregnancy was excellent and it was similar to that reported in singleton pregnancy. However, due to small number of trisomy 13 cases, accurate assessment of the efficacy of NIPT for trisomies 13 could not be achieved.

\section{MZ Female's Self- and Co-Twin Assessment: An Actor- Partner Interdependence Model}

M. Egorova, Yu Chertkova and O. Parshikova

Lomonosov Moscow State University, Moscow, Russia

The Actor-Partner Interdependence Model (APIM) provides methodological framework to describe the mutual influences between the dyad members. Theoretically it based on the recognition of the dependency between dyad members, and mathematically it is a method for evaluating variance, both between dyads and within dyads. In our study we used APIM, based on structural equation modeling (SEM). There were evaluated the actor and partner effects in MZ female twin pairs in self and co-twin assessment. Our study involved 49 twin pairs aged from 18 to 64 years old (mean age was 27 years). Unipolar scales of semantic differential (45 adjectives) were used for actual self and co-twin assessments as well as the desired changes in personality in oneself and in co-twin. According to exploratory factor analysis we obtained 5 factors: Agreeableness, Activity, Perseverance/Conscientiousness, Ascendancy/ Proneness to Conflict and Sensitivity. For all factors escape Ascendancy/ Proneness to Conflict only actor effects were founded between actual and desired self-assessments. Ascendancy/ Proneness to Conflict demonstrated partner and actor effects for actual and desired selfassessments. The relations between self and co-twin (actual assessment and desired changes) are more complex. The results are interpreted in the context of the role of the shared and nonshared environment in the variability of personality traits of twins.

\section{Factors Associated With Care-Seeking for Low Back Pain When Genetics and the Familial Environment are Considered}

Thomas G. Patterson ${ }^{1}$, Ana Paula Carvalho E Silva ${ }^{1}$, , Aquino ${ }^{1}$, Manuela Ferreira ${ }^{2}$ and Paulo Ferreira ${ }^{1}$

${ }^{1}$ Department of Physiotherapy, Faculty of Medicine and Health, The University of Sydney, NSW, Australia and ${ }^{2}$ Institute of Bone and Joint, Kolling Institute, Faculty of Medicine and Health, University of Sydney, NSW, Australia

Background: Low back pain (LBP) is the leading cause of disability worldwide. Inappropriate and ineffective care-seeking for LBP causes substantial economic burden to governments and the healthcare system. Objective: To investigate lifestyle and health-related factors associated with care-seeking (including pain medication use) in individuals experiencing LBP, after controlling for important genetic and early environmental confounders through the use of a withintwin pair case-control design. Design: A secondary analysis of observational longitudinal data, derived from the Australian Twin low BACK pain (AUTBACK) study, was performed on 66 twin pairs who presented with similar symptoms of LBP at baseline but became discordant for care-seeking behaviour over one month. Methods: Subjective and objective assessment of pain intensity, disability, depression, sleep quality, physical activity and body mass index were performed. Data were analyzed using stepwise conditional logistic regression in two stages: within-pair case control for monozygotic and dizygotic twins together; and within-pair case-control analysis of monozygotic twins only. Results were expressed as odds ratios $(O R)$ and 95\% confidence intervals (CI). Results: Higher LBP intensity (OR 2.9; 95\% CI $[1.3,6.8])$ and poorer sleep quality (OR 10.9; $95 \%$ CI $[1.5,77.7])$ were the main factors that increased the likelihood of care-seeking for LBP. These associations remained significant and increased in magnitude after adjusting for genetic confounding. Conclusions: Individuals with higher LBP intensity and worse sleep quality are more likely to seek care for LBP, and this relationship is likely to be causal after adjustment of familial and genetic confounding.

\section{Do Parents Propagate Income Inequality among Children? Evidence from Chinese and Swedish Twins}

Aiday Sikhova ${ }^{1}$, Sven Oskarssonq ${ }^{2}$ and Rafael Ahlskog ${ }^{2}$

${ }^{1}$ The Ohio State University, Columbus, OH, USA and ${ }^{2}$ Uppsala University, Uppsala, Sweden

In this paper we provide comprehensive evidence on the role of parents in driving within-family income inequality by considering the dynamic nature of parent-child relationships that span children's entire lives. Specifically, we examine three important mechanisms through which parents may impact the children: investment in education, inter-vivos transfers, and bequests. Since parental investment and transfer behaviors may depend on their country of residence, to assess generalizability of our estimates, we focus the analysis on families from China and Sweden - that are different in the level of socioeconomic development and demographic characteristics. Accounting for endogeneity of parental behaviors, we find that Chinese parents tend to reinforce income inequality between the children using inter-vivos transfers: a $10 \%$ increase in a twin child's salary at the time of transfer increases inter-vivos transfers by $8.7 \%$. Swedish parents, on the other hand, tend to invest, transfer, and bequeath equal amounts to both children. Given considerable differences between Chinese and Swedish parents in their attitudes towards within-family income inequality, we demonstrate that these differences can be largely explained by differences in parental education levels in two countries. This, in turn, emphasizes that, accounting for the level of parental education in the target population, our findings can be generalized to other settings.

\section{The Association Between Saving Disposition and Financial Distress: A Genetically Informed Approach}

Alexandros Giannelis ${ }^{1}$, Emily A. Willoughby ${ }^{1}$, Robin Corley $^{2}$, Christian Hopfer $^{2}$ John Hewitt ${ }^{2}$, Scott Vrieze ${ }^{1}$, James J. Lee ${ }^{1}$ and Matt McGue ${ }^{1}$

${ }^{1}$ University of Minnesota, Minneapolis, MN, USA and ${ }^{2}$ University of Colorado, Boulder, CO, USA

The causes of variation in exposure to economic hardship have remained largely unexplored. Saving disposition has been shown 
to be associated with financial distress, but the association could be driven by other economic factors, behavioral traits or even genetic effects. Using a sample of 2,522 American twins, we develop measures of saving disposition and financial distress. We estimate the association between the two scales controlling for income, as well as cognitive and personality traits. We also estimate the proportion of covariance between the traits that is due to genetic effects, and use a sibling comparison design to rule out genetic or environmental confounding. Our results suggest that there is a causal association between saving disposition and financial distress, although the direction of causation remains unclear.

\section{Monozygotic Twin Similarities for AD Biomarkers: The Twin60++ Study}

Anouk den Braber ${ }^{1,2}$, Jori Tomassen ${ }^{2}$, Emma M. Coomans ${ }^{2,4}$, Elles Konijnenberg ${ }^{2}$, Mara ten Kate ${ }^{2}$, Rik Ossenkoppele ${ }^{2,5}$, Betty M. Tijms ${ }^{2}$, Charlotte E. Teunissen ${ }^{3}$, Frederik Barkhof ${ }^{4}$, Bart N. M. van Berckel ${ }^{4}$, Philip Scheltens ${ }^{2}$, Dorret I. Boomsma ${ }^{1}$, Eco J.C. de Geus ${ }^{1}$ and Pieter Jelle Visser ${ }^{2,6,7}$

${ }^{1}$ Department of Biological Psychology, Vrije Universiteit Amsterdam, Amsterdam, the Netherlands, ${ }^{2}$ Alzheimer Center Amsterdam, Department of Neurology, Amsterdam Neuroscience, Vrije Universiteit Amsterdam, Amsterdam UMC, Amsterdam, The Netherlands,

${ }^{3}$ Neurochemistry Laboratory Department of Clinical Chemistry, Amsterdam Neuroscience, Vrije Universiteit Amsterdam, Amsterdam UMC, Amsterdam, The Netherlands, ${ }^{4}$ Department of Radiology \& Nuclear Medicine, Amsterdam Neuroscience, Vrije Universiteit Amsterdam, Amsterdam UMC, Amsterdam, The Netherlands, ${ }^{5}$ Clinical Memory Research Unit, Lund University, Lund, Sweden, ${ }^{6}$ Alzheimer Center Limburg, School for Mental Health and Neuroscience, Maastricht University, Maastricht, The Netherlands and ${ }^{7}$ Department of Neurobiology, Care Sciences and Society, Division of Neurogeriatrics, Karolinska Institutet, Stockholm, Sweden

Background: There is limited information on the contribution of genes and environment to early Alzheimer's disease (AD) pathophysiology. In a longitudinal cohort of cognitively normal older monozygotic twins we estimated twin resemblance for amyloid- $\beta$ and tau aggregation markers, assessed etiology of comorbidity among markers, and tested their relation with subsequent memory decline. Methods: The PreclinAD-Twin60++ study started in 2014 $(N=204$; age:70.8 \pm 7.8$) ; 58 \%$ female). Twin correlations were estimated for amyloid- $\beta$ and tau aggregation as measured with $[18 \mathrm{~F}]$ flutemetamol and [18F]flortaucipir positron emission tomography scans and in cerebrospinal fluid (CSF). Etiology of association among markers was explored by cross-twin cross-trait and intra-pair difference analyses. Associations of AD biomarkers with memory decline (3 assessments in 6 years) was tested using linear mixed models. Results: Twins correlated .50-.52 for amyloid- $\beta$ and $0.56-0.64$ for tau aggregation markers. In CSF, lower (i.e., more abnormal) amyloid- $\beta$ levels were associated with higher (i.e., more abnormal) tau levels $(\beta=-0.54, p<.001)$ and this relation held while ruling out possible genetic confounding in intra-pair difference analysis $(\beta=-0.53, p<.001)$. Also, amyloid- $\beta$ levels in a twin could predict tau levels in the co-twin $(r=-.38, p<.001)$. Both amyloid- $\beta$ and tau levels at baseline predicted subsequent memory decline (amyloid- $\beta *$ time: $\beta=0.04, \quad p<.001$; tau*time: $\beta=-0.03$, $p=.008)$. Conclusions: The moderate to high twin resemblance for amyloid- $\beta$ and tau, the robust association between markers, and their predictive value towards memory change in cognitively normal individuals, show that very early $\mathrm{AD}$ pathophysiology is influenced by both genetic and environmental factors and underpin the use of these markers for defining the risk for clinical AD.

\section{Food Intake and Epigenetic Alteration of the Spermatozoa of Twins (FEAST): Understanding the Contribution of Diet on the Sperm Epigenome}

Jessica M. Preston ${ }^{1}$, Victoria George ${ }^{2}$, Yen Ting Wong ${ }^{2}$, Robert McLachlan ${ }^{2}$, Jeffrey M. Craig ${ }^{2}$ and Romain Barrès ${ }^{1}$

${ }^{1}$ Novo Nordisk Foundation Center for Basic Metabolic Research; University of Copenhagen, Copenhagen, Denmark and ${ }^{2}$ School of Medicine; Deakin University, Melbourne, VIC, Australia, ${ }^{3} \mathrm{Hudson}$ Institute of Medical Research, Melbourne, VIC, Australia

Our lifestyle affects the quality and the epigenetic patterning of sperm cells, which may in turn influence health outcome of our children. However, the specific effect of a man's diet has yet to be fully appreciated. We have initiated the FEAST study to determine how current dietary trends are shaping the epigenome of sperm cells, providing a snapshot of the diet-associated epigenetic marks which may be involved in epigenetic inheritance. In this study, we will be providing healthy male twin pairs with discordant diets for a period of three weeks, wherein diets represent a highly processed diet and an unprocessed diet. These diets mimic what males in industrialized countries are currently actually eating, in comparison to what it is presently recommended that they should eat. Both prior to and following administration of these diets, biological samples, including semen samples, anthropometric characteristics, and survey information will be collected. We will compare parameters using a co-twin randomised design, in order to elucidate the differential epigenetic patterning resulting from the differential dietary groups. This study will provide essential information on the human-specific impact of diet on the epigenetic characteristics of spermatozoa, which will provide insight into how diet in fathers may impact child health, along with aiding in the future development of more robust dietary guidelines for fathers-to-be.

\section{Postmenopausal Hormone Therapy and Dementia}

Laura Ekstrøm Løkkegaard ${ }^{1,2,3}$, Mikael Thinggaard ${ }^{1,2,3}$, Marianne Nygaard ${ }^{1,2,3}$, Jesper Hallas ${ }^{4}$, Merete Osler ${ }^{5}$ and Kaare Christensen ${ }^{1,2,3}$

${ }^{1}$ Unit of Epidemiology, Biostatistics and Biodemography, Department of Public Health, University of Southern Denmark, Odense, Denmark, ${ }^{2}$ The Danish Twin Registry, Department of Public Health, University of Southern Denmark, Odense, Denmark, ${ }^{3}$ Danish Aging Research Center, Unit of Epidemiology, Biostatistics and Biodemography, Department of Public Health, University of Southern Denmark, Odense, Denmark, ${ }^{4}$ Clinical Pharmacology, Pharmacy and Environmental Medicine, Department of Public Health, University of Southern Denmark, Odense University Hospital, Odense, Denmark and ${ }^{5}$ Center for Clinical Research and Prevention, Bispebjerg and Frederiksberg Hospitals, Denmark

Introduction: Dementia risk is higher for women than men, potentially due to hormonal factors. The role of postmenopausal hormone therapy (HT) in dementia risk is unclear. Observational studies have previously found a protective effect on dementia whereas most clinical studies, and some observational studies, have found increased dementia risk following use of systemic HT. Our aim was to investigate the association between HT and dementia using the unique structure of Danish health registries in a nested case-control study and a co-twin control design which controls for familial confounding and shared genetics. Methods: We identified through Danish national registries from 1995 to 2011: (a) 2700 female singletons with incident dementia and 13,492 matched controls; (b) 290 female twins with incident dementia and a non-demented co-twin; c) HT use and education. Data were analyzed using conditional logistic regression and McNemar's $\chi^{2}$-test. Results: The odds-ratio between systemic HT and dementia was 1.05, $95 \%$ CI $[0.93,1.19]$ in singletons and $2.10,95 \%$ CI $[0.99,4.46])$ in twins. A statistically significant association was found for systemic HT used 
solely before 2003 in both populations, however no dose-response association was found. Conclusion: Using Danish nationwide registries and controlling for education and for familiar factors in a subsample, systemic HT was associated with increased dementia risk if used before 2003. We found no dose-response association, indicating that results could be due to confounding by indication, as HT was more commonly prescribed before 2003 or too short a follow-up, though causality cannot be ruled out.

\section{Etiological and Comorbidity Factors of Internalizing and Externalizing Symptoms in a Nonclinical Sample of Children and Adolescents: A Twin Study}

Stefano de Francesco ${ }^{1}$, Simona Scaini ${ }^{1}$, Emanuela Medda ${ }^{2}$, Laura Camoni ${ }^{2}$, Maria A. Stazi ${ }^{2}$ and Corrado Fagnani ${ }^{2}$

${ }^{1}$ Child and Youth Lab, Sigmund Freud University, Milan, Italy and ${ }^{2}$ Centre for Behavioural Sciences and Mental Health, Istituto Superiore di Sanità, Rome, Italy

Introduction: The co-occurrence between internalizing and externalizing symptoms is an extremely recurrent phenomenon. The frequency with which this comorbidity occurs raised the hypothesis that behind both domains there may be a common susceptibility represented by a general psychopathology factor ('p factor'). However, it is still unclear whether this common liability is a stable phenomenon, or whether it is affected by age-related variations. Methods: Data on internalizing (divided into fear and distress subdimensions) and externalizing symptoms of 803 twin pairs enrolled in the Italian Twin Register were collected through the DSM-oriented scales of CBCL/6-18. The sample was stratified into two age groups: 6-14 (365 pairs) and 15-18 (438 pairs) years. Model-fitting analysis was performed to detect, in each subsample, the model that best described the genetic and environmental bases of the phenotypic correlation among the symptoms. Results: In both age groups, moderate to high phenotypic correlations among fear, distress and externalizing symptoms were found. Model-fitting analysis showed that, for the 6-14 group, a Cholesky model best fitted the data, whereas for the 15-18 age group, the best explanation of the data was provided by a common pathway model showing that nearly $50 \%$ of total variance of each trait was due to common genetic factors. Conclusions: Our results support a common susceptibility behind internalizing and externalizing symptoms, mainly genetic in origin, which becomes more evident at the beginning of puberty. From a clinical standpoint, these results would suggest to monitor externalizing risk in the presence of internalizing symptoms (or vice versa) at both individual and familial level.

\section{Familial Risk and Heritability of Hematologic Malignancies in the Nordic Twin Study of Cancer}

Signe B. Clemmensen ${ }^{1}$, Jennifer R. Harris ${ }^{2}$, Jonas Mengel-From ${ }^{1,3}$, Wagner H. Bonat ${ }^{4}$, Henrik Frederiksen ${ }^{5,6}$, Jaakko Kaprio ${ }^{7}$ and Jacob v. B. Hjelmborg ${ }^{1}$

${ }^{1}$ Department of Epidemiology, Biostatistics, and Biodemography, Institute of Public Health, University of Southern Denmark and Danish Twin Registry, Institute of Public Health, University of Southern Denmark, Odense, Denmark, ${ }^{2}$ Division of Health Data and Digitalisation, Norwegian Institute of Public Health, Oslo, Norway, ${ }^{3}$ Department of Clinical Genetics, Odense University Hospital, Odense, Denmark, ${ }^{4}$ Department of Statistics, Paraná Federal University, Curitiba, PR, Brazil, ${ }^{5}$ Department of Haematology, Odense University Hospital, Odense, Denmark, ${ }^{6}$ Department of Clinical Research, Institute of Public Health, University of Southern Denmark, Odense, Denmark, and ${ }^{7}$ Department of Public Health and Institute for Molecular Medicine Finland, University of Helsinki, Helsinki, Finland

We aimed to explore the genetic and environmental contributions to variation in the risk of hematologic malignancies and characterize familial dependence within and across hematologic malignancies. The study base included 316,397 individual twins from the Nordic Twin Study of Cancer with a median of 41 years of follow-up: 88,618 (28\%) of the twins were monozygotic, and 3,459 hematologic malignancies were reported. We estimated the cumulative incidence by age, familial risk, and genetic and environmental variance components of hematologic malignancies accounting for competing risk of death. The lifetime risk of any hematologic malignancy was $2.5 \%$ (95\% CI $[2.4,2.6])$, as in the background population. This risk was elevated to $4.5 \%$ (95\% CI $[3.1,6.5])$ conditional on hematologic malignancy in a dizygotic co-twin and was even greater at $7.6 \%(95 \% \mathrm{CI}[4.8,11.8])$ if a monozygotic co-twin had a hematologic malignancy. Heritability of the liability to develop any hematologic malignancy was $24 \%(95 \%$ CI $[14,33])$. This estimate decreased across age, from approximately $55 \%$ at age 40 to about $20-25 \%$ after age 55 , when it seems to stabilize. In this largest ever studied twin cohort with the longest follow-up, we found evidence for familial risk of hematologic malignancies. The discovery of decreasing familial predisposition with increasing age underscores the importance of cancer surveillance in families with hematological malignancies.

\section{Determinants of Age at Menarche: A Study in the California Twin Program}

Maryam Salehi ${ }^{1}$, Amie E. Hwang ${ }^{2}$, Wendy Cozen $^{1}$ and Thomas M. Mack ${ }^{2}$

${ }^{1}$ University of California, Irvine, CA, USA and ${ }^{2}$ University of Southern California, Los Angeles, CA, USA

Background: Age at menarche (AAM) is determined by both heritable and nonheritable factors; the variation due to genetic factors has been estimated at 50 to $80 \%$. If the negative consequences of extreme variations in age at menarche are to be subject to intervention, resolution of this inconsistency is required. We aimed to evaluate the risk factors for earlier menarche in discordant pairs for AAM (defined as at least 6 months difference in AAM in paired twins) and determinants of early menarche ( $\leq 11$ years) in concordant pairs. Methods: We included selfreported data from 11,781 female pairs from California Twin Program and conducted analyses of comparisons between and within pairs (Falconer method, Chi-square, and multivariable logistic regression). Results: $42.7 \%$ of all pairs were concordant, included $56.9 \%$ of monozygotic (MZ) and $28.9 \%$ of dizygotic (DZ) pairs. Heritable, shared environmental and unshared environmental factors contributing to AAM were estimated to be $68 \%, 7 \%$ and $39 \%$ respectively. The MZ twin who grew faster during puberty was more likely to have earlier menarche than her co-twin (OR 11.6, 95\% CI [8.4, 15.8]). The taller twin tended to have the later menarche (OR 0.44, 95\% CI [0.32, 0.62]). More exercise decreased the likelihood of earlier menarche by $30 \%$ (OR 0.70 [95\% CI [0.53, 0.93]). Non-white pairs with less-educated mothers and lower levels of education tended to have early menarche $(p<.05)$. Conclusions: Heritability explains $68 \%$ of AAM. Twins who reported relatively more exercise during past year than co-twins had later menarche, suggesting that even minor differences in exercise can have an effect.

\section{Prenatal Exposure to Tobacco Smoke is Associated with Placental Biomarkers of Aging}

Esmée M. Bijnens ${ }^{1,2}$, Catherine Derom ${ }^{2}$, Evert Thiery ${ }^{3}$, Eline Meireson ${ }^{2}$, Steven Weyers ${ }^{2}$ and Tim S. Nawrot ${ }^{1,4}$

${ }^{1}$ Centre for Environmental Sciences, Hasselt University, Diepenbeek, Belgium, 2Department of Humans Structure and Repair, Ghent University Hospital, Ghent, Belgium, ${ }^{3}$ Department of Neurology, Ghent University Hospital, Ghent, Belgium and ${ }^{4}$ Department of Public Health, Leuven University (KU Leuven), Leuven, Belgium

Introduction: Aging is a very complex biological phenomenon deriving from an interaction between genetic and environmental factors. 
A known accelerator of the aging process is the smoke of cigarettes. As aging starts before birth, we hypothesize that prenatal exposure to tobacco smoke is negatively associated with placental biomarkers of aging. Methods: The East Flanders Prospective Twin Survey is a population based register of multiple births in the Belgium started in 1964 and collecting placental tissue at birth. Telomere length and mitochondrial DNA content were measured in placental tissue of children participating in a follow-up study on intelligence. Data on maternal smoking during pregnancy was collected retrospectively. Results: Our results show that mothers who smoked during pregnancy have a significant lower mitochondrial DNA content in placental tissue compared to nonsmokers and also compared to mothers who smoked before pregnancy. In addition to this, we found that an increase in the number of cigarettes smoked a day during pregnancy is associated with a decrease in placental mitochondrial DNA content. Conclusion: These preliminary results indicate show an inverse association between maternal smoking during pregnancy and mitochondrial DNA content. This suggests that in utero exposure to tobacco smoke might accelerate aging and has implications on health outcomes later in life.

\section{The Long-Term Effects of Selective Fetal Growth Restriction in MONochorionic twins: The LEMON Study}

Sophie Groene ${ }^{1,2}$, Arno A. W. Roest ${ }^{3}$, Christiaan de Bruin ${ }^{4}$, Ilja de Vreede ${ }^{5}$, Bas T. Heijmans ${ }^{2}$, Enrico Lopriore ${ }^{1}$ and Jeanine M. M. van Klink ${ }^{1}$

${ }^{1}$ Neonatology, Department of Pediatrics, Leiden University Medical Center, Leiden, the Netherlands, ${ }^{2}$ Molecular Epidemiology, Department of Biomedical Data Sciences, Leiden University Medical Center, Leiden, the Netherlands, ${ }^{3}$ Pediatric Cardiology, Department of Pediatrics, Leiden University Medical Center, Leiden, the Netherlands, ${ }^{4}$ Pediatric Endocrinology, Department of Pediatrics, Leiden University Medical Center, Leiden, the Netherlands and ${ }^{5}$ Pediatric Pulmonology, Department of Pediatrics, Leiden University Medical Center, Leiden, the Netherlands

Introduction: Selective fetal growth restriction (sFGR) in monochorionic (MC) twin pregnancies is characterized by a large intertwin growth discrepancy due to unequal placental sharing. Neonatal morbidity and mortality associated with sFGR have been thoroughly described, but data on long-term outcome is lacking although we know that fetal growth restriction (FGR) in singletons has been associated with an increased risk of adverse health outcomes. Therefore, the main objective of the LEMON study is to assess long-term neurodevelopmental, cardiovascular, pulmonary, and growth outcomes in a cohort of MC twins with sFGR and to compare outcomes within twin pairs. Materials and methods All MC twin pairs with sFGR and without other MC twin pathology born in our centre between 2002 -2017 are eligible for inclusion. We defined sFGR as a birth weight discordance $\geq 20 \%$. To assess long-term neurodevelopmental outcome, cognitive and motor development will be evaluated using standardized psychometric age-appropriate tests and a neurological examination. Echocardiography will be used to assess differences in structural cardiac measures and cardiac function, including aortic pulse-wave velocity (aPWV) and carotid intima-media thickness (cIMT). Spirometry will be recorded in children $\geq 4$ years old to quantify lung function, including forced vital capacity (FVC), forced expiratory volume in one second (FEV1) and forced mid-expiratory flow rate $(\mathrm{FEF}(25-75 \%)$. Growth will be assessed using available childhood growth curves from the primary care system and by detailed anthropometric measurements. Results: The study started in January 2021. So far, we have included approximately 20 twin pairs (30\% of the anticipated population) aged 10-17 years of which we have collected data on neurodevelopmental outcome, childhood growth patterns and pubertal status. These preliminary results will give us a first glimpse of any within-pair differences between the smaller and larger twin in long-term outcome. Conclusions: By studying a cohort of sFGR MC twins, we will gain more insight into the long-term consequences of FGR. We believe that with our expertise in both follow-up studies and complicated MC twins, we will be able to provide strong evidence on long-term neurodevelopment, cardiovascular health, lung function and childhood growth patterns of sFGR MC twins and thereby aid in future counselling, early identification of children at risk and devising feasible management and treatment options.

\section{Changes in Structural Brain Development After Selective Fetal Growth Restriction in Monochorionic Twins}

Sophie G. Groene ${ }^{1,3}$, Linda S. de Vries ${ }^{1}$, Femke Slaghekke ${ }^{2}$, Monique C. Haak ${ }^{2}$, Bas T. Heijmans ${ }^{3}$, Christiaan de Bruin ${ }^{4}$, Arno A. W. Roest ${ }^{5}$, Enrico Lopriore ${ }^{1}$, Jeanine M. M. van Klink ${ }^{1}$ and Sylke J. Steggerda ${ }^{1}$

${ }^{1}$ Division of Neonatology, Willem-Alexander Children's Hospital, Department of Pediatrics, Leiden University Medical Center, Leiden, the Netherlands, ${ }^{2}$ Division of Fetal Therapy, Department of Obstetrics, Leiden University Medical Center, Leiden, the Netherlands, ${ }^{3}$ Division of Molecular Epidemiology, Department of Biomedical Data Sciences, Leiden University Medical Center, Leiden, the Netherlands, ${ }^{4}$ Division of Pediatric Endocrinology, WillemAlexander Children's Hospital, Department of Pediatrics, Leiden University Medical Center, Leiden, the Netherlands and ${ }^{5}$ Division of Pediatric Cardiology, Willem-Alexander Children's Hospital, Department of Pediatrics, Leiden University Medical Center, Leiden, the Netherlands

Objectives: Fetal growth restriction (FGR) may permanently alter brain development resulting in lifelong structural and functional changes. However, in studies addressing this research question, FGR singletons are primarily compared to matched appropriately grown singletons, a design that is inherently biased by differences in genetic and maternal factors. To overcome these limitations, we conducted a within-pair comparison of structural cerebral measurements in identical twin pairs discordant for fetal growth. Methods: Structural cerebral measurements on neonatal cerebral ultrasound were compared between the smaller twin and larger twin of monochorionic twins with selective fetal growth restriction (sFGR), defined as a birth-weight discordance $\geq 20 \%$, born in our center between 2010-2020. Each twin pair was also matched to an appropriately grown singleton based on sex and gestational age at birth. Results: We included 58 twin pairs with sFGR, with a median gestational age at birth of 31.7 (IQR 29.9 -33.8) weeks and a median birth weight for the smaller twin and the larger twin of respectively 1155 grams versus 1725 grams (median birth weight discordance of 32\%). The smaller twin had significantly smaller cerebral structures (corpus callosum, vermis, cerebellum), white/deep gray matter and intracranial surface and volume. Intracranial volume discordance and birth-weight discordance correlated significantly $(\mathrm{r}=0.443, \mathrm{p}=0.004)$. Intracranial volume discordance was smaller as opposed to birth-weight discordance ( $19 \%$ vs. $32 \%$ respectively, $p<.0001)$. After correction for intracranial volume, all observed differences (except for biparietal diameter) ceased to exist. Conclusions: sFGR in monochorionic twins is associated with an overall, proportional restriction in brain growth on neonatal cerebral ultrasound for the smaller twin, in line with previous singleton studies. The amount of birth-weight discordance translates into a discordance in the size of brain structures as well, albeit smaller as opposed to the amount of birth-weight discordance. 


\section{Creating Multiple Connections: Exploring Experiences of Families With Twins, Triplets or More}

Katie McLeod-Peters ${ }^{1}$, Nathalie Turville ${ }^{2}$, Liz Hingley ${ }^{1}$ and Jane Denton ${ }^{1,3}$

${ }^{1}$ Elizabeth Bryan Multiple Births Centre, Birmingham City University, Birmingham, UK, ${ }^{2}$ School of Nursing and Midwifery, Birmingham City University, Birmingham, UK and ${ }^{3}$ Multiple Births Foundation, Queen Charlotte's and Chelsea Hospital, London, UK

Introduction: Adjusting to family life and caring for twins, triplets or more presents psychosocial, practical and financial challenges for the parents. There is limited research into the lived experiences of multiple birth families during the early years and their perceptions of family life with young multiples. The aim of the study is to explore and describe the parenting journey of parents of multiples from pregnancy to starting school through the use of photographs. The study was led by the Elizabeth Bryan Multiple Births Centre, Birmingham City University. Team members include a professional photographer/researcher and a parent of twins. Methods: An exploratory qualitative study was undertaken using photo-elicitation. This was chosen to help parents of multiples explore subjects and express emotions, which are often difficult to put into words. Families with multiples aged 4-6 in the West Midlands, UK, were invited to participate in in-depth interviews conducted on-line. The interviews were structured around parental choice of 10 significant photographs providing insight into their unique experiences as multiple birth families. Results: Photo-elicitation proved both a meaningful research method and cathartic process for participants. The study is still in progress so initial key findings will be described. Conclusions: The use of photographs enabled the researchers to literally 'see' the world and the situation through the eyes of the participants Further understanding of the moments that illustrate the 'highs' and 'lows' of family life with young multiples, will contribute to providing greater insight into the support needed by multiple birth families.

\section{Supporting Multiple Birth Families; Establishing an Evidence Base to Inform Health Visitor Practice}

Nathalie Turville ${ }^{1}$, Lara Alamad ${ }^{1}$, Jane Denton ${ }^{2}$ and Merryl Harvey ${ }^{1}$

${ }^{1}$ School of Nursing and Midwifery Elizabeth Bryan Multiple Births Centre, Birmingham City University, Birmingham, UK and ${ }^{2}$ Multiple Births Foundation, Queen Charlotte's and Chelsea Hospital, London, UK

Introduction: As parents adjust to family life and caring for multiples, they are faced with many social, emotional, practical and economic challenges. The transition to parenthood and the early years are widely recognised as critical time periods that influence longer term outcomes of infants and their families. The role of health visitors (HV) in supporting multiple birth families is essential however there is limited research in to this area. The aim of the study was to establish an evidence base of health visitor experiences and perceptions supporting multiple birth families. Method: Health Visitors were invited to complete an on-line survey. The questionnaire consisted of closed questions, Likert scales and open questions to capture participant experiences. Descriptive statistical analysis was used quantitative data and thematic analysis for open questions. Results: Most HVs had twins on their current workload. Most had not received any specific training regarding needs of multiple birth families. Supporting the families within the confines of reduced time and increased workload was challenging. Daily tasks of caring for multiples (e.g. breast-feeding, weaning and managing crying) were the main areas HVs and parents wanted more information about.
Conclusion: In the UK, HVs are uniquely positioned to support multiple birth families. However, the findings suggest that many HVs are aware that care and support provided multiple birth families falls short of meeting their needs due to gaps in education and workload demands. It is recommended that strategies to facilitate HV support for multiple birth families should be explored and implemented.

\section{Global Applied Learning Experience Project: A Mixed-Method Study Protocol}

Shayesteh Jahanfar ${ }^{1}$, Shannon Lynch ${ }^{1}$, Patel $^{1}$ and Mojgan Gharipour ${ }^{2}$

${ }^{1}$ Tufts School of Medicine, Department of Public Health and Community Medicine, Boston, MA, USA and ${ }^{2}$ Division of Genetics and Epigenetics Studies, Cardiovascular Research Institute, Isfahan University of Medical Sciences, Isfahan, Iran

Introduction: All diseases are either entirely genetic, environmental, or, most often, a combination of the two. Separating the genetic and the environmental contributions to disease conditions is important for both preventative and treatment options as it helps identify the risk factors for developing certain diseases. Twin studies are unique in that they allow us to study the interaction between an individual's genes and their environment by matching for confounders such as age, race, intra-uterine environment, and early childhood environment. Method: Through this Global Applied Learning Experience we aim to achieve an understanding of the challenges and benefits of establishing and maintaining a global twin registry. To better understand the interaction between anthropometric variables and risk factors in anxiety and depression among twins and to explore the clustered relationship that is seen throughout the Isfahan Twin Registry (ITR) dataset. Methodology: This is a mixedmethod study of twins registered at Isfahan Twin Registry (ITR). Established in 2017, one of the youngest twin registries across the globe, ITR is a pilot study to establish a nationwide twin registry in Iran and aims to obtain comprehensive information about complex diseases and their risk factors from twins and multiples living in Isfahan. This registry continued to collect data and recruited participants for a longitudinal study to determine five-year incidence of cardiovascular disease, cancer and diabetes. A major advantage of the twin cohort study design is the ability to study multiple outcomes that can be associated with a single exposure or multiple exposures in a single study. Master of Public Health curriculum at Tufts is a unique program that encourages global reach to enrich students' exposure to international collaboration, provide opportunity to practice public health in supportive and supervised settings and culminate an academically based experience. As a part of Global Applied Learning Experience at Tufts, we reached out to ITR to establish a research-training activity, aiming to gain skill sets required by $\mathrm{CEPH}$ accreditation for the MPH program. Results: This project will begin with a literature review of twin studies. We intend to attend the 19th International Twin Conference to enrich our understanding of twin studies. Equipped with the background information of how twin registries are established across the globe, we will design a list of structured questions to conduct the qualitative part of our study. We will then communicate with the management team at ITR to interview ten stakeholders about challenges and feasibility of creating a Population-Based National Twin Registry in a Middle East Country. We also intend to identify individuals responsible for establishing twin registries at global level, obtain their contact information during the conference and interview them. The individual interviews will probe attitudes, beliefs, desires, and experiences in establishing a twin registry. Due to COVID-19 status, the interviews will take place virtually. We will then generate major themes from the narrative interviews and use the framework method to systematically identify categories and themes identified by reading the transcripts. We will find relevant quotations supporting the 
credibility of data selecting the proper theme. For the quantitative part of our study, we will work on collected data of adults at the ITR. The hypothesis is to (1) obtain risk factors associated with anxiety/depression among twins, and (2) acquire the relationship between anthropometric variables and anxiety/depression in twins. We will conduct a secondary data analysis using cluster analysis to ensure twins correlation is taken into account. We will build two predictive regression models, one for each specific objectives. We will then prepare knowledge translation pieces to share with scientific communities as well as the public. Conclusion: This study protocol is designed for ALE program using twin data at global level.

\section{Zygosity and Sex Based Cardiometabolic Risk Factors in Isfahan Twin Registry}

Mojgan Gharipour, Minoo Dianatkhah and Nizal Sarrafzadegan

Isfahan Cardiovascular Research Center, Cardiovascular Research Institute, Isfahan University of Medical Sciences, Isfahan, Iran

Background: The Isfahan Twin Registry was initiated in late 2017 by ascertainment of twins born since 1960 in Isfahan, Iran to provide a unique source for large studies on both genetic and environment influence on the incidence of noncommunicable disease, aging and age-related diseases. Study population recruitment: Twins are invited and the details of the registry were announced in broadcast programs as well as in social media. Information on demographics and socioeconomic status, lifestyle behaviors such as food consumption, smoking habit, physical activity, psychological health, medical records, pharmacological treatment, surgeries, or any para-clinical tests were collected by validated questionnaires. Zygosity was determined using an 8-item questionnaire. Weight, height and waist circumference and, blood pressure were measured by trained nurses. Whole blood, serum, plasma, buffy coat, stool, guttery papers, nail, hair, and urine samples were collected. DNA has been collected from whole blood for the all the ITR participants. Preliminarily results: Over the two years, a total of 224 monozygotic and 586 dizygotic pairs in all age groups were registered at the ITR. The number of registered participants based on age category till November 2020 was 96 infant's pairs $(1-24$ months), (monozygotic $=14$, dizygotic $=82$ ), 230 early childhoods, late childhoods, adolescent pairs (2-18) $($ monozygotic $=148$, dizygotic $=418)$, and adult pairs (monozygotic $=62$, dizygotic $=82$ ). Our findings show no significant difference between monozygotic infants on birth weight and birth height. But the birth weight of boy-boy dizygotic infants is slightly higher than boy-girl and girl-girl twins. Among early childhood dizygotic twins (2-6 years old), triglyceride level is higher in boy-boy ( $p=.01$ ). Boy-boy monozygotic adolescence twin (13-18 years) have higher waist circumference than girl-girl monozygotic twin $(82.1 \pm$ 10.6 vs. $71.4 \pm 5.6, p=.013$ ). In the monozygotic adults' twin group, the mean of waist circumference, systolic blood pressure, triglyceride, aspartate aminotransferase, and alanine aminotransferase were higher among boy-boy monozygotic pairs. Among dizygotic adult twins, the mean age of girl-boys was higher than boy-boy, girl-girls (35.7 \pm 10.1 years, vs. $30.8 \pm 9.1$, and $30.8 \pm 9.1$, respectively). There was no significant difference between body mass index, waist circumference, fasting blood sugar, total cholesterol, HDL and LDL-cholesterol, triglyceride, aspartate aminotransferase, and alanine aminotransferase $(p>.05)$ in adult dizygotic twin. Conclusion: This registry provides both clinical and epidemiological data and help us to have information on biological processes, we may be able to discover biological pathways and design new preventive and therapeutic strategies for diseases.

\section{Heritability of Sleep Quality Across the Adult Age Span: Analysis in Two Mediterranean Samples}

Juan J. Madrid-Valero ${ }^{1}$, Juan F. Sánchez-Romera ${ }^{2}$, Corrado Fagnani ${ }^{3}$,

Emanuela Medda ${ }^{3}$, Maria A. Stazi ${ }^{3}$, Virgilia Toccaceli ${ }^{3}$ and Juan R. Ordoñana

${ }^{1}$ University of Alicante, Alicante, Spain, ${ }^{2}$ University of Murcia, Murcia, Spain and ${ }^{3}$ Istituto Superiore di Sanità, Rome, Italy

Introduction: Sleep quality worsens markedly with age and most of the sleep problems usually show increasing prevalence and intensity after adulthood. Our main objective was to compare the genetic and environmental influences on sleep quality in two samples from different Mediterranean countries. Additionally, we aimed to analyze the role of age as a possible moderating factor on the heritability of adult sleep quality. Method: The sample comprised 5865 subjects (42.0\% female), aged 18-93 years $($ mean $=47.3)$, participating in the Murcia Twin Registry and the Italian Twin Registry. Sleep quality was measured using the Pittsburgh Sleep Quality Index. Univariate and moderation twin analyses were performed using structural equation models. Results: Heritability of sleep quality in the total sample was estimated at $36 \%$ $(95 \%$ CI $[28,43])$, with a significant effect of genetic factors (AE model). Similar estimates were found in both samples (in both cases the best fitting model was an AE model). The heritability of sleep quality was estimated at 31\% (95\% CI $[24,38])$ in the Spanish sample and at 39\% (95\% CI $[28,46])$ in the Italian sample. Preliminary moderation analyses showed that heritability estimates remain stable across the age span in the global sample. Discussion: Our results are consistent with previous studies and meta-analyses which find a moderate genetic influence on sleep quality. This genetic influence is of a similar magnitude in two samples from different Mediterranean countries. Results also show that such influence appears to remain stable across the life span when considering the whole sample.

\section{Explorative Study of the Epigenetics of Brain and Central Nervous System Cancers in a Population Based Sample of Twins}

Asmus Skovgaard $^{1}$, Hans Christian Petersen ${ }^{2}$, Kaare Christensen ${ }^{1,3,4}$, Jacob Hjelmborg ${ }^{1}$ and Mette Soerensen ${ }^{1,4}$

${ }^{1}$ Epidemiology, Biostatistics and Biodemography, Department of Public Health, University of Southern Denmark, Odense C, Denmark, ${ }^{2}$ Data Science and Statistics, Department of Mathematics and Computer Science, University of Southern Denmark, Odense C, Denmark, ${ }^{3}$ Department of Clinical Biochemistry and Pharmacology, Odense University Hospital, Odense C, Denmark and ${ }^{4}$ Department of Clinical Genetics, Odense University Hospital, Odense C, Denmark

Introduction: Cancer is a leading cause of morbidity and mortality worldwide. Brain and central nervous system (CNS) cancers are especially detrimental, due to low survival probabilities or potential neurological complications. In recent years, epigenetics has been suggested to be central for cancer development, interestingly however, genomewide epigenetic studies of these cancers are scarce. With the aim to identify molecular markers of brain and CNS cancers, we performed an explorative study of the epigenetics of these cancers in monozygotic twins. Methods: Genomewide DNA methylation data were available for 1016 Danish monozygotic twins, comprising 508 twin pairs, in the age range 30-90 years. Thirteen individuals had a diagnosis for brain and CNS cancers according to the Danish Patient Registry, while their co-twin had no such diagnosis. Two epigenomewide association analyses were performed: a linear mixed-effects regression model exploring all 1016 individuals, and a linear regression model investigating the intrapair differences 
in epigenetics in the 13 cancer discordant twin pairs. Results: Thirtyseven genes were identified by analysis of all 1016 individuals, while two other genes were observed by the intrapair analysis. The most prominent genes were DIRAS3 for the former, and CASZ1 for the latter. Both are tumor suppressor genes, known from among others, glioma and neuroblastoma. Conclusions: This study suggests several candidate genes of potential relevance for brain and CNS cancers. Although additional studies are needed for external validation, the study indicates that the application of twin populations could be promising for identifying molecular markers of human traits and disease.

\section{Twin Tempos: Cross-Correlations of Young Twins' Social and Emotional Skills}

Lisabeth F. DiLalla

Southern Illinois University School of Medicine, Carbondale, IL, USA

Introduction: Impaired emotion skills, especially recognizing or expressing fear and anger, may lead to poor peer interactions. Genetic risk for badly expressing fear has been related to increased victimization. Thus, early identification of children at risk for poor emotion skills is important. This study hypothesized that preschoolers who respond with inappropriate aggression or noncompliance to story stems would have poorer emotion skills at school age, and this relationship would be genetically mediated. Methods: Fiveyear-old twins were asked to complete stories; responses were rated as prosocial, aggressive, passive, or noncompliant. Children returned to the lab at age 6-13 years old and completed two tests of emotion skills: the DANVA for emotion recognition, where they reported faces as looking happy, sad, fearful, or angry; and a task where they were told to show each of those expressions and coders later rated their photos for emotion expression accuracy. Results: Preschoolers who provided ineffective story responses (e.g., noncompliance or aggression in ambiguous scenarios) showed some poorer emotion skills later, especially for fear. Cross-twin correlations showed that some of these relationships have a genetic component, with a larger cross-trait correlation for MZ twins than DZ twins for the relationship between ineffective story completions and later poor recognition of fear faces. Conclusion: Preschoolers with poor story completion skills may show weaker emotion skills later, putting them at risk for negative peer interactions such as being victimized. Shared genes may explain some of these relationships, but difficult early peer interactions may also be a risk factor.

\section{Tracing Human Twinning Process Using Somatic Mutations}

Christopher J. Yoon ${ }^{1,2}$, Chang Hyun $\mathrm{Nam}^{1}$, Eun Saem Choi ${ }^{3}$, Sol Hee Bang ${ }^{4}$, Soo A. Oh ${ }^{1}$ Seung Mi Lee ${ }^{3}$, Young Mi Jung ${ }^{3}$, Marij Gielen ${ }^{5}$, Catherine Derom ${ }^{6}$, Malachi Griffith ${ }^{2}$, Obi L. Griffith ${ }^{2}$, Jong Kwan Jun ${ }^{3}$, Joohon Sung ${ }^{7}$ and Young Seok Ju ${ }^{1,4}$

${ }^{1}$ Graduate School of Medical Science and Engineering, Korea Advanced Institute of Science and Technology, Daejeon, Korea, ${ }^{2}$ Department of Genetics, Washington University School of Medicine, St. Louis MO, USA, ${ }^{3}$ Department of Obstetrics and Gynecology, Seoul National University College of Medicine, Seoul, Korea, ${ }^{4}$ Genome Insight Inc. Daejeon, Korea, ${ }^{5}$ Department of Complex Genetics, Maastricht University, Maastricht, the Netherlands, ${ }^{6}$ Department of Obstetrics and Gynaecology, Ghent University, Ghent, Belgium and ${ }^{7}$ Department of Epidemiology, School of Public Health, Seoul National University, Seoul, Korea

Introduction: Although monozygotic twins are derived from a single zygote, their genome sequences are slightly different due to somatic mutations that accumulated during the early embryogenesis. Using the early embryonic mutations (EEMs) as cellular barcodes, it is now possible to trace clonal dynamics in the monozygotic twinning by delineating the relative contribution of each embryonic cell in the twin development. Here, we investigated the patterns of EEMs between monozygotic twins with information of their chorionicity and amnionicity. Methods: We recruited 7 monochorionic monoamniotic (MCMA), 7 monochorionic diamniotic (MCDA), and 4 dichorionic diamniotic (DCDA) monozygotic twin families. Whole-genome (60x for twins, 30x for parents) and targeted deep sequencing of the parents and monozygotic twins were conducted for discovering and validating EEMs. Results: From the genome sequences of 18 twin families, we identified hundreds of EEMs. A substantial proportion of the EEMs were unequally present between twins. The segregation pattern of the mutations in twin individuals was associated with the chorionicity and the amnionicity of the twins: (1) MCMA twins mostly shared their EEMs which indicates their late twinning event; (2) In contrast, DCDA twins had EEMs that were exclusively present in one twin, suggesting their early separation; (3) MCDA twins showed a variable mutational pattern that suggested more heterogeneity in the twinning event. Conclusions: EEMs allowed us to reconstruct the early cellular dynamics during the twinning process with approximate embryonic cell stages in molecular time. Our results support the hypothesis that the chorionicity and amnionicity of the monozygotic twins are determined by the timing of twinning events during embryogenesis.

\section{NONAGINTA Study: Cognition in 90-Year-Old Twins}

Eero Vuoksimaa

Institute for Molecular Medicine Finland (FIMM), HiLIFE, University of Helsinki, Helsinki, Finland

Introduction: The oldest-old - those who are 90 years or older are the fastest growing population segment in many countries. However, we know very little about cognitive aging of this age group. There is a need for population-based studies to investigate predictors of cognitive impairment and antecedents of successful cognitive aging in the oldest-old. Assessment of cognition via telephone is a cost-effective approach in epidemiological studies and in screening of cognitive status at individual level. However, the utility of cognitive telephone assessment in the oldest-old is not known. Methods: We have established NONAGINTA memory and health in 90-year-olds - study to investigate cognitive functioning and related health factors in the oldest-old. We use two validated telephone interviews to measure cognitive performance. Participants are from a population-based older Finnish Twin Cohort study with baseline questionnaire in 1975. This design offers a unique opportunity to study health and health-related behaviors in relation to old age cognition with more than 45 years of follow-up. Results: Ongoing data collection started in 2020 and to date there are 118 twins who have answered to questionnaire and 67 twins who have participated in telephone interview assessing cognitive functioning. Preliminary data on cognitive functioning and health related factors will be presented. Conclusions: Telephone interview is a feasible procedure to assess cognitive functioning in the oldest-old. These data will make it possible to study risk and protective factors of cognitive impairment in the oldest-old. Telephone screening makes it possible to invite participants in more detailed in-laboratory measurements. 


\section{Utilizing Birthweight Discordant Monozygotic Twins to Investigate the Effect of the In Vivo Early Embryonic Environment on DNA Methylation}

Rebekka Koeck ${ }^{1,2}$, Eline Meireson ${ }^{3}$, Catherine Derom ${ }^{3}$, Steven Weyers ${ }^{3}$, Han Brunner ${ }^{1,4}$ Aafke van Montfoort ${ }^{5}$, Masoud Zamani Esteki ${ }^{1,2}$ and Marij Gielen ${ }^{6}$

${ }^{1}$ Department of Clinical Genetics, Maastricht University Medical Centre+, Maastricht, the Netherlands, ${ }^{2}$ Department of Genetics and Cell Biology, GROW School for Oncology and Developmental Biology, Maastricht University, Maastricht, the Netherlands, ${ }^{3}$ Department of Obstetrics and Gynaecology, Ghent University Hospital, Ghent University, Ghent, Belgium, ${ }^{4}$ Department of Human Genetics, Radboud University Medical Center, Nijmegen, the Netherlands, ${ }^{5}$ Department of Obstetrics and Gynaecology, GROW School for Oncology and Developmental Biology, Maastricht University Medical Center+, Maastricht, the Netherlands and ${ }^{6}$ Department of Epidemiology and Nutrition and Toxicology Research Institute Maastricht (NUTRIM), Maastricht University Medical Centre, Maastricht, the Netherlands

Introduction: Human embryos undergo epigenetic (DNA methylation) reprogramming during pre-implantation development. Although the environmental impact on this phenomenon can be studied in the context of in vitro fertilization (IVF) procedures, little is known about the interplay between the in vivo environment and epigenetic reprogramming as in vivo conceived human embryos cannot be sampled. Nonetheless, it has been established that peri-conception insults can induce methylome alterations identifiable in adults. Therefore, longitudinal studies are needed to establish the persistence of epigenetic marks from conception throughout life and to interpret their clinical significance. Monozygotic twins uniquely allow the study of the early embryonic environment as their environment can be considered identical until the moment of splitting, after which they can experience microenvironment differences. Study design: Participants consist of the 120 most birthweight discordant monozygotic adult twin pairs, stratified by placentation (monochorionic, dichorionic separate placentas, dichorionic fused placentas), from the East Flanders (Belgium) prospective Twin Survey (EFPTS). Placenta biopsies and birth records were collected prospectively at birth while saliva samples and health information will be collected prospectively. Reduced representation bisulfite sequencing (RRBS) will be used to profile the methylome. Placental intrapair methylation differences, birthweight discordance and placental morphology associations, as well as the persistence of epimutations (saliva) and their relation to adult outcomes will be analysed. Results: Since recruitment commenced (July 2021), 49 individuals have agreed to participate and 28 have completed the questionnaire ( $n=25$ without missing data). Imminently, saliva samples will be requested from twin pairs who have completed the questionnaire.

\section{Prenatal Exposure to Tobacco Smoke is Associated With Placental Biomarkers of Aging}

Esmée M. Bijnens ${ }^{1,2}$, Catherine Derom² ${ }^{2}$ Evert Thiery ${ }^{2}$, Eline Meireson ${ }^{2}$, Steven Weyers ${ }^{2}$ and Tim S. Nawrot ${ }^{1,3}$

${ }^{1}$ Centre for Environmental Sciences, Hasselt University, Diepenbeek, Belgium, ${ }^{2}$ Ghent University Hospital, Ghent University, Ghent, Belgium and ${ }^{3}$ Laboratory of Respiratory Diseases and Thoracic Surgery (BREATHE) Leuven University, Leuven, Belgium

Introduction: Aging is a very complex biological phenomenon deriving from an interaction between genetic and environmental factors. A known accelerator of the aging process is the smoke of cigarettes. As aging starts before birth, we hypothesize that prenatal exposure to tobacco smoke is negatively associated with placental biomarkers of aging. Methods: The East Flanders Prospective Twin Survey is a population-based register of multiple births in Belgium that started in 1964 and collected placental tissue at birth. Telomere length and mitochondrial DNA content were measured in placental tissue of children participating in a follow-up study on intelligence. Data on maternal smoking during pregnancy were collected retrospectively. Results: Our results show that mothers who smoked during pregnancy have a significant lower mitochondrial DNA content in placental tissue compared to nonsmokers and also compared to mothers who smoked before pregnancy. In addition to this, we found that an increase in the number of cigarettes smoked a day during pregnancy is associated with a decrease in placental mitochondrial DNA content. Conclusion: These preliminary results indicate show an inverse association between maternal smoking during pregnancy and mitochondrial DNA content. This suggests that in utero exposure to tobacco smoke might accelerate aging and has implications on health outcomes later in life.

\section{Study in Heritability of MRI White Matter Hyperintensities in Twins}

Amirreza Alijanpourotaghsara ${ }^{1}$, David Strelnikov ${ }^{1}$, Laszlo Szalontai ${ }^{1}$, Zsofia Jokkel ${ }^{1}$, Piroska Marton ${ }^{1}$, Bianka Forgo ${ }^{1,2}$, Csaba Olah $^{3,4}$, Laszlo Kostyal ${ }^{4}$, David L Tarnoki ${ }^{1}$ and Adam D. Tarnoki ${ }^{1}$

${ }^{1}$ Medical Imaging Centre, Semmelweis University, Budapest, Hungary, ${ }^{2}$ Department of Radiology, Faculty of Medicine and Health, Örebro University, Örebro, Sweden, ${ }^{3}$ Department of Neurosurgery, University of Debrecen, Debrecen, Hungary and ${ }^{4}$ Department of Radiology, Borsod County University Teaching Hospital, Miskolc, Hungary

Introduction: White matter hyperintensities $(\mathrm{WMH})$ indicate white matter brain lesions in magnetic resonance imaging (MRI), which can be used as a marker for brain aging, cerebrovascular and neurodegenerative disorders. Aim: This study aimed to evaluate the genetic components of WMH in a healthy twin population. Methods: 172 healthy adult twins from the Hungarian Twin Registry (104 monozygotic, MZ, and 68 dizygotic, DZ pairs; mean age $52.6 \pm 13.4$ years) underwent brain MRI, including fluid attenuation inversion recovery (FLAIR) sequences. We investigated the total number, juxtacortical, infratentorial, and cerebellar counts of the WMH lesions. Age and sex-adjusted MZ and DZ correlations were calculated, and the univariate ACE model was applied. Results: Age and sex adjusted MZ intrapair correlations were higher than DZ correlations indicating strong genetic influence in each lesions $(r \mathrm{MZ}=.781$, $r \mathrm{DZ}=.554$ for total count; $r \mathrm{MZ}=.515, r \mathrm{DZ}=.430$ for juxtacortical count; $r \mathrm{MZ}=.671, r \mathrm{DZ}=.379$ for infratentorial count; and $r \mathrm{MZ}=.743, r \mathrm{DZ}=$ .205 for cerebellar count), indicating a strong heritability ( $A=82.8 \%$, $A=57.8 \%, A=68.7 \%$ and $A=72.5 \%$, respectively). The rest of the variance was influenced by unique environmental effects ( $\mathrm{E}$ between $17.2 \%$ and $42.2 \%$, respectively). Conclusions: WMH lesion count is strongly influenced by genetic effects, especially in the infratentorial region. Further studies should investigate whether lifestyle interventions could influence this substantial heritability through epigenetic modification.

\section{Comparison of Circle of Willis Variants in Monozygotic and Dizygotic Twins}

David Strelnikov ${ }^{1}$, Amirreza Alijanpourotaghsara ${ }^{1}$, Laszlo Szalontai $^{1}$, Zsofia Jokkel ${ }^{1}$, Piroska Marton ${ }^{1}$, Bianka Forgo, Csaba Olah ${ }^{3,4}$, Laszlo Kostyal ${ }^{4}$, David L. Tarnoki ${ }^{1}$ and Adam D. Tarnoki ${ }^{1}$

${ }^{1}$ Medical Imaging Centre, Semmelweis University, Budapest, Hungary, ${ }^{2}$ Department of Radiology, Faculty of Medicine and Health, Örebro University, Örebro, Sweden, ${ }^{3}$ Department of Neurosurgery, University of Debrecen, Debrecen, Hungary and ${ }^{4}$ Department of Radiology, Borsod County University Teaching Hospital, Miskolc, Hungary

Introduction: The Circle of Willis (CoW) is thought to play a key role in providing compensatory blood supply to the brain in times of 
ischemia. Morphological variants of this anastomosis are frequently observed in healthy individuals. Aim: The purpose was to assess the proportion of variance attributable to genetic and environmental factors for CoW morphological variants. Methods: 124 adult healthy twins from the Hungarian Twin Registry (42 monozygotic, MZ and 20 dizygotic, DZ pairs, mean age $49.8 \pm 15.3$ years) underwent time-of-flight magnetic resonance angiography. We investigated the variants of the anterior and posterior CoW according to morphological categories as well as the diameters of the anterior and posterior communicating arteries (ACoA, PCoA), and A1 and P1 segments of the anterior and posterior cerebral arteries (ACA, PCA), respectively. Age and sex-adjusted $\mathrm{MZ}$ and $\mathrm{DZ}$ correlations were calculated. Results: Complete CoW was found in $51 \%$ while as incomplete CoW was observed in $49 \%$ of subjects. Anterior and posterior variants of CoW were found in $35 \%$ and in $68 \%$, respectively. DZ intrapair correlations were higher than $\mathrm{MZ}$ correlations indicating no genetic influence $(r \mathrm{MZ}=.174, r \mathrm{DZ}=.23$ for $\mathrm{ACoA}$ diameter; $r \mathrm{MZ}=.267, r \mathrm{DZ}=.389$ for right $\mathrm{PCoA}$ diameter; $r \mathrm{MZ}=.249$, $r \mathrm{DZ}=.307$ for left $\mathrm{PCoA}$ diameter; $r \mathrm{MZ}=0.032, r \mathrm{DZ}=.368$ for anterior $\mathrm{CoW}$ variants; $r \mathrm{MZ}=.169, r \mathrm{DZ}=.533$ for posterior $\mathrm{CoW}$ variants; $r \mathrm{MZ}=.632, r \mathrm{DZ}=.714$ for complete CoW; $r \mathrm{MZ}$ $=.489, r \mathrm{DZ}=.615$ for incomplete $\mathrm{CoW})$. Conclusion: $\mathrm{CoW}$ variants seem to be influenced by environmental factors in healthy individuals. Further investigations may identify potential environmental factors affecting these variants.

\section{Differences in DNA Methylation-Based Aging Within Twin Pairs Discordant for Cancer}

Hannes Frederik Bode, Aino Heikkinen, Jaakko Kaprio and Miina Ollikainen

Helsinki University, Institute for Molecular Medicine, Helsinki, Finland

DNA methylation-based aging prediction associates with cancer, with both cancer tissue and blood showing increased epigenetic age acceleration (eAA). It is unknown to what extent twins from pairs discordant for cancer differ in their eAA. We aimed to investigate whether eAA associates with cancer risk in these twins and thus serves as a putative biomarker for cancer risk. We identified 47 monozygotic and 48 same-sex-dizygotic cancer discordant twin pairs in the Finnish Twin Cohort study with blood samples available cross-sectionally between 17 years before and 31 years after the diagnosis. We first performed pan cancer analyses (95 pairs), then separately for breast cancer (24 pairs) and all the other cancers (71 pairs). Within-pair differences in eAA were identified by t-test, and cross-sectional associations between time-to-diagnosis and within-pair differences in eAA were assessed by linear regression. Twins from pairs sampled before cancer diagnosis did not differ for eAA. The within-pair differences in eAA before cancer diagnosis increased significantly the closer the cancer diagnosis was, and this acceleration extended to years after the diagnosis (all cancers, 1.9; breast-cancer, 2.1; all other than breast-cancer, 1.7 month increase per year). Pairs sampled after the diagnosis differed for eAA across all cancers ( 2 years 5.8 months), in breast-cancer (3 years 3.5 months), and in all other cancer (2 years 1.8 months). The results suggest that eAA associates with the cancer diagnosis itself. This may be due to the epigenetic alterations in relation to the cancer, its treatment or diagnosis associated lifestyle changes.

\section{Continuity of Genetic Risk for Aggression Across the Life-Course}

Camiel M. van der Laan ${ }^{1,2}$, José J. Morosoli-Garci凶?á ${ }^{3}$, Steve G. A. van de Weijer ${ }^{2}$, Lucía Colodro-Conde ${ }^{3}$, ACTION Consortium, Michelle K. Lupton ${ }^{3}$, Brittany L. Mitchell ${ }^{3}$, Kerrie McAloney ${ }^{3}$, Richard Parker ${ }^{3}$, Jane M. Burns ${ }^{4}$, Ian B. Hickie ${ }^{5}$, René Pool ${ }^{1}$, Jouke-Jan Hottenga ${ }^{1}$, Nicholas G. Martin ${ }^{3}$, Sarah E. Medland ${ }^{3}$, Michel G. Nivard ${ }^{1}$ and Dorret I. Boomsma ${ }^{1}$

${ }^{1}$ Biological Psychology, Vrije Universiteit, Amsterdam, the Netherlands, ${ }^{2}$ The Netherlands Institute for the Study of Crime and Law Enforcement, Amsterdam, the Netherlands, ${ }^{3}$ Department of Genetics and Computational Biology, QIMR Berghofer Medical Research Institute, Brisbane, QLD, Australia, ${ }^{4}$ Faculty of Health Sciences, The University of Sydney, Sydney, Australia and ${ }^{5}$ Brain and Mind Centre, University of Sydney, Sydney, NSW, Australia

Introduction: We test whether genetic influences that explain individual differences in aggression in early life also explain individual differences across the life-course. Methods: In two cohorts from The Netherlands $(N=13,471)$ and Australia $(N=5,628)$, polygenic scores (PGSs) were computed based on a genomewide meta-analysis of childhood/adolescence aggression. In a novel analytic approach, we ran a mixed-effects model for each age (Netherlands: 12-70 years, Australia: 16-73 years), with observations at the focus age weighted as 1 , and decaying weights for ages further away. We call this approach a 'rolling weights' model. Results: In the Netherlands, the estimated effect of the PGS was relatively similar from age 12 to age 41 , and decreased from age 41 to 70 . In Australia, there was a peak in the effect of the PGS around age 40 years. Conclusion: These results are a first indication from a molecular genetics perspective that genetic influences on aggressive behavior that are expressed in childhood continue to play a role later in life.

\section{Influences of Parental Warmth and Discipline on Five- Year-Old Twins' Intrapair Cooperation}

Riley L. Marshall ${ }^{1}$, Rachel Weisbecker ${ }^{1}$ and Lisabeth Fisher DiLalla ${ }^{2}$

${ }^{1}$ Department of Psychology, Southern Illinois University, Carbondale, IL, USA and ${ }^{2}$ Family and Community Medicine, Southern Illinois University School of Medicine, Carbondale, IL, USA

Introduction: Cooperation and conflict are intrinsic to twin relationships. MZ twins may exhibit more cooperation and less conflict than do DZ twins, and recent research has associated parenting with parent-reported twin conflict. However, associations between observed parenting and observed twin cooperation have not been examined. Therefore, we investigated twin zygosity and parenting as predictors of twin cooperation. We hypothesized that 1) MZ twins would exhibit more cooperation than DZ twins, and 2) twins who experienced more positive parenting would engage in more cooperation. Methods: Participants were 187 same-sex 5-year-old twins (94 MZ, $93 \mathrm{DZ}$ ) participating in an ongoing twin study. Data were taken from a parent-child interaction task in which the twins and one parent played with puzzles for 10 minutes. Parental warmth and discipline were coded separately for each child. Additionally, children's cooperation was coded for each minute in which the children interacted. Results: MZ twins were not significantly more cooperative than DZ twins $(p=.488)$. Multilevel linear modeling regressions showed a between but not within family effect for increased parental warmth related to increased child cooperation $(\mathrm{p}=.030)$. Also, 
increased parental discipline significantly predicted decreased child cooperation $(\mathrm{p}<.001)$, and there was significant within pair variance ( $p=.035$ ) but not a significant effect of shared genes between parent discipline and child cooperation $(p=.295)$. Conclusions: Results suggest that parental behaviors that include more warmth and less discipline are associated with increased cooperation in twins. Neither of these relationships are due to shared genes, supporting the importance of shared environment for early child cooperation.

\section{Educational Influences on Cognitive Aging: Exploring Unequal Opportunities Through Genetic Propensities and Societal Change}

Maria Ericsson ${ }^{1,2}$, Nancy Pedersen ${ }^{1}$ and Miriam Mosing ${ }^{1,2,3}$

${ }^{1}$ Karolinska Institutet, Stockholm, Sweden, ${ }^{2}$ University of Melbourne, Melbourne, VIC, Australia and ${ }^{3}$ Max Planck Institute, Munich, Germany

Introduction: This project aimed to disentangle genetic and environmental factors underlying the impact of education on cognitive health in late life. We explored the relative protective effects of (1) a genetic propensity for education (PGSEdu), and (2) actual attained education on cognitive health, and their interplay, taking into account the impact of social policy changes that advance gender and class equality. Methods: Data were retrieved from the The Screening Across the Lifespan of Twins (SALT). Data included participants (born between 1886 and 1958) with available genotype data $(n=15,004)$. Cognitive ability was measured via the TELE. Analyses included: investigating familial confounding in the relationship between attained education and cognitive ability, using the polygenic scores to investigate genetic influence on cognition, applying withinfamily estimation to the genetic analyses to investigate prGE. To investigate how a discordance between PGSEdu and attained education was related to late life health, two different approaches were applied both using residuals and extreme groups of discordance (and concordance). Results: Our analyses show that both attained education and measured genetic propensities for education are independently related to cognitive ability in late life, with higher genetic propensities and higher level of education predicting better cognitive ability. Discordance analyses further supported the importance of attained education as a predictor for cognitive ability. Conclusions: These results strengthens the causal influence of education on late-life cognitive ability but also emphasizes the importance of familial confounding and independent influence of genetic propensities.

\section{Twins and Causal Inference: Leveraging Nature's Experiment}

Frühling V. Rijsdijk ${ }^{1}$, Tom A. McAdams ${ }^{1,2}$, Helena M.S. Zavos ${ }^{3}$ and Jean-Baptiste Pingault ${ }^{1,4}$

${ }^{1}$ Social, Genetic and Developmental Psychiatry Centre, Institute of Psychiatry, Psychology and Neuroscience, King's College London, London, UK, ${ }^{2}$ Promenta Research Centre, University of Oslo, Oslo, Norway, ${ }^{3}$ Department of Psychology, Institute of Psychiatry, Psychology \& Neuroscience, King's College London, London, UK and ${ }^{4}$ Clinical, Educational and Health Psychology, Division of Psychology and Language Sciences, University College London, London, UK

We discuss how samples comprising monozygotic and dizygotic twin pairs can be used for the purpose of strengthening causal inference by controlling for shared influences on exposure and outcome. The basic twin model used to inform the biometric decomposition of population variance into genetic, shared environmental, and nonshared environmental influences can be extended to explore whether associations between exposure and outcome survive correction for shared etiology (common causes). We review several analytical approaches that can be applied to twin data for this purpose. These include multivariate structural equation models, cotwin control methods, direction of causation models (cross-sectional and longitudinal), and extended family designs used to assess intergenerational associations. We conclude by highlighting some of the limitations and considerations that researchers should be aware of when using twin data for the purposes of interrogating causal hypotheses.

\section{Mental Health Impact of COVID-19: A Longitudinal Study on Italian Twins}

Emanuela Medda, Corrado Fagnani, Angelo Picardi, Antonella Gigantesco,

Maurizio Ferri, Cristina D'Ippolito and Maria A. Stazi

Centre of Reference for Behavioural Sciences and Mental Health, Instituto Superiore di Sanità, Rome, Italy

Introduction: There are concerns that the Covid-19 crisis and measures to mitigate virus transmission may have caused a considerable burden on population mental health. In June 2020, the Italian Twin Registry (ITR) set up a longitudinal study to investigate the impact of Covid-19 on depressive and anxiety symptoms. Methods: In June 2020 and December 2020 (Wave1 and Wave2, respectively) twins were invited to complete an online questionnaire. The questionnaire included the Patient Health Questionnaire (PHQ-9), and the sixitem version of the State-Trait Anxiety Inventory (STAI-6) for detecting probable depression and anxiety, respectively. For each of the two instruments, phenotypic correlation between the two waves was estimated, and bivariate Cholesky models were fitted to explore the genetic and environmental contributions to the observed longitudinal patterns. Results: About 300 adult twin pairs participated in the study. Substantial longitudinal correlations were observed for PHQ-9 $(r=.67)$ and STAI-6 scores $(r=.57)$. Bivariate models including additive genetic and unshared environmental influences best fitted the longitudinal data for these traits. Under these models, heritability estimates for the PHQ-9 were 0.38 (Wave1) and 0.43 (Wave2), and 0.40 (Wave1) and 0.38 (Wave2) for the STAI-6. Genetic correlations between the two waves (.99 PHQ-9, .91 STAI-6) were higher than unshared environmental correlations (.45 PHQ-9, .36 STAI-6). Conclusions: The study suggests that the pandemic led to an increase in depressive and anxiety symptoms. The longitudinal stability of this symptomatology is mainly genetically determined, while the observed environmental discontinuity is consistent with changes in life conditions during the pandemic.

\section{Study of Twin Pedigrees to Investigate Mechanisms of Male Microchimerism in Females}

Brandon N. Johnson ${ }^{1,2}$, Henrike E. Peters ${ }^{3,4}$, Cornelis B. Lambalk ${ }^{3,4}$, Conor V. Dolan ${ }^{2}$, Gonneke Willemsen ${ }^{2}$, Lannie Ligthart ${ }^{2}$, Velja Mijatovic ${ }^{3}$, Jouke-Jan Hottenga ${ }^{2}$, Erik A. Ehli ${ }^{1}$ and Dorret I. Boomsma ${ }^{2,4}$

${ }^{1}$ Avera Institute for Human Genetics, Avera McKennan Hospital \& University Health Center, Sioux Falls, SD, USA, ${ }^{2}$ Netherlands Twin Register, Department of Biological Psychology, Vrije Universiteit, Amsterdam, The Netherlands, ${ }^{3}$ Department of Reproductive Medicine, Amsterdam UMC, Vrije Universiteit, Amsterdam, the Netherlands and ${ }^{4}$ Amsterdam Reproduction and Development (AR\&D) Research Institute, Amsterdam UMC, Vrije Universiteit, Amsterdam, the Netherlands

Introduction: Natural chimerism, cells originating from two or more zygotes, has most commonly been identified as microchimerism 
( $<1 \%$ minor cell population) resulting from fetomaternal exchange during pregnancy. However, male microchimerism also occurs among females with no history of pregnancy suggesting alternative risk factors. To provide more insight, we investigated patterns and prevalence of male microchimerism within twin families. Methods: We included data from 446 adult female participants from two generation twin pedigrees recruited by the Netherlands Twin Register (NTR). Biobanked DNA extracted from peripheral blood was tested via quantitative real-time PCR for a Y chromosome specific gene, DYS14, relative to measure of a common beta-globin gene. Using chi-squared, generalized estimating equations and logistic regression procedures, we tested whether male microchimerism was more prevalent when having a male co-twin, older brother, or male offspring. Results: Male microchimerism was detected in $26.9 \%$ of participants. Pedigree characteristics revealed male microchimerism was not explained by having a male co-twin, older brother, or male offspring. Similarity was observed between the correlations of monozygotic twin-pairs and first-degree relatives. Male microchimerism detection was positively associated with age $(p=.02)$, while outliers with the greatest concentrations of male microchimerism presented with a significantly lower age $(p=.034)$. Conclusions: This study did not support our hypothesis that a particular pedigree trait, such as having a male co-twin, promotes male microchimerism in women. However, these findings do suggest that additional characteristics such as time or molecular traits may be of significant interest for understanding the prevalence of microchimerism.

\section{Genetic Covariances Underlying Associations Between Mathematics, Reading and Working Memory}

Zhixin Zhu and Lee A. Thompson

Case Western Reserve University, Cleveland, USA

Working memory plays an important role in reading and math performance. However, whether the relationships between working memory, reading and arithmetic are influenced by verbal and/or visuospatial working memory remains unclear. The current study examined genetic and environmental effects on individual differences in the associations between reading, math and different components of working memory, and employed longitudinal twin data to trace the trajectories of their relationships across development. Twin data were drawn from the Western Reserve Reading and Math Project, an ongoing longitudinal twin project from Ohio. Reading ability was assessed using Word Identification, Word Attack, and Passage Comprehension subtests from the Woodcock Reading Mastery Test (WRMT); math ability was assessed using Calculation, Applied Problem, Quantitative Concepts, and Fluency subtests from the Woodcock-Johnson III Achievement test; visuospatial working memory was measured using the Flag test from the Factor Reference Cognitive Test and the Corsi Block test; verbal working memory was measured with the StanfordBinet Intelligence Scale Memory for Digits Subtest. Our results showed moderate to substantial correlations among all measures, with a decrease in the association between visuospatial working memory and each reading measure across development.
Univariate genetic analysis demonstrated that genetic influences contributed to individual differences in both early and later reading performance. On the other hand, genetic effects did not strongly affect individual differences in math ability at the younger age, but accounted for a significant proportion as the twins got older.

\section{Isfahan Twins Cohort Study: A Longitudinal Follow Up Based on Twins Registry}

Nizal Sarrafadegab, Nizal Sarrafzadegan and Mojgan Gharipour

Isfahan Cardiovascular Research Center, Cardiovascular Research Institute, Isfahan University of Medical Sciences, Isfahan, Iran

Background and Aim: The Isfahan twins Registry (ITR) started in 2017 and was performed on twins, multiples, and their parents. Its aims were to obtain comprehensive information on noncommunicable diseases (NCD) and their behavioral and metabolic risk factors and to develop a biobank for future genetic and epigenetic studies. A year later, we decided to follow all recruited twins in a prospective longitudinal cohort (ITC) to determine the 5-year incidence of some NCDs, such as cardiovascular disease, cancers and diabetes, and their risk factors. Methods: All enrolled 1000 twins were followed up and re-evaluated every year as Isfahan Twin Cohort (ITC) study. The enrolment into the ITR was all-inclusive without any exclusion criteria. After obtaining consent forms, we completed questionnaires on demographics, twins' similarities, lifestyle behaviors, medical history and performed complete physical examination. Participants provided blood and bio samples including (whole blood, serum, plasma, buffy coat, stool, nail, hair, and urine) Anthropometric measurements and blood pressure were measured by trained health professionals. Blood samples were analyzed for hematology, fasting blood sugar, total cholesterol, low- and high-density lipoproteins, aspartate aminotransferase, alanine aminotransferase, and quantitative C-reactive protein. Extra blood and other bio samples were stored in -80 freezers. Results: Until October 2020, a total of 1000 participants consisted of 395 twins, 62 triples, 3 quadruple and 2 more were registered. Finally, two persons were excluded due to lack of collaboration of the second pair. The number of registered participants based on age category were 82 infants (1-24 month), 364 early childhood (2-7 years), 294 late childhood (7-12 years), 102 adolescences (13-18 years) and 156 adults $(\geq 19)$. Our primary findings show no significant difference between males and females according to their weight, height, waist and hip circumferences in early childhood. However, in late childhood, females have higher LDL, triglyceride, and total cholesterol than males. The mean level of triglyceride and total cholesterol in late childhood females and males, were 96.22 \pm 48.46 vs. $81.42 \pm 29.86(p=.002), 159.99 \pm 26.08$ vs. $148.42 \pm 23.85$ $(p<.001)$, respectively. In adolescents, the mean level of fasting blood sugar in females and males were $84.98 \pm 7.5$ vs. $89.71 \pm$ $6.76)$, respectively, $(p=.004)$. Conclusion: The ITR then ITC studies created rich source of data that can be used in future genetic and epigenetic studies on NCDs and their risk factors. Twins studies specifically cohorts with prospective follow ups are helpful to dissect disease mechanisms in humans, and to design new preventive and therapeutic strategies for diseases 


\section{The Babytwins Study Sweden (BATSS): A Multi-Method Infant Twin Study of Genetic and Environmental Factors Influencing Infant Brain and Behavioral Development}

Terje Falck-Ytter ${ }^{1,2,3}$, Linnea Hamrefors ${ }^{1,2}$, Monica Siqueiros Sanches ${ }^{2,4}$, Ana Maria Portugal ${ }^{2}$, Mark Taylor $^{5}$, Danyang Liं ${ }^{2,6}$, Charlotte Viktorsson ${ }^{1}$, Irzam Hardiansyah ${ }^{2}$, Lynnea Myers ${ }^{2,7,8}$, Lars Westberg ${ }^{9}$, Sven Bölte ${ }^{2,10,11}$, Kristiina Tammimies ${ }^{2,6}$ and Angelica Ronald ${ }^{12}$

${ }^{1}$ Development and Neurodiversity Lab (DIVE), Department of Psychology, Uppsala University, Uppsala, Sweden, ${ }^{2}$ Center of Neurodevelopmental Disorders (KIND), Centre for Psychiatry Research; Department of Women's and Children's Health, Karolinska Institutet and Stockholm Health Care Services, Stockholm, Sweden, ${ }^{3}$ Swedish Collegium for Advanced Study (SCAS), Uppsala, Sweden, ${ }^{4}$ Center for Interdisciplinary Brain Sciences Research, Department of Psychiatry and Behavioral Sciences, Stanford University, Stanford, CA, USA, ${ }^{5}$ Department of Medical Epidemiology \& Biostatistics, Karolinska Institutet, Stockholm, Sweden, ${ }^{6}$ Astrid Lindgren Children's Hospital, Karolinska University Hospital, Stockholm, Sweden, ${ }^{7}$ Department of Nursing, Gustavus Adolphus College, St. Peter, MN, USA, ${ }^{8}$ Clinical Pediatrics, Department of Women's and Children's Health, Karolinska Institutet, Stockholm, Sweden, ${ }^{9}$ Department of Pharmacology, Institute of Neuroscience and Physiology at the Sahlgrenska Academy, University of Gothenburg, Gothenburg, Sweden, ${ }^{10} \mathrm{Child}$ and Adolescent Psychiatry, Stockholm Health Care Services, Stockholm, Sweden, ${ }^{11}$ Curtin Autism Research Group, Curtin School of Allied Health, Curtin University, Perth, WA, Australia and ${ }^{12}$ Department of Psychological Sciences, Birkbeck, University of London, London, UK

Twin studies have long contributed to the understanding of the relative contributions of genes and environment to phenotypic trait variation, including cognitive and brain activation measures. However, in terms of methodologies such as electroencephalography (EEG) and eye-tracking, key methods in developmental cognitive and social neuroscience, infant twin studies are almost non-existent. We will describe the Babytwins Study Sweden (BATSS), a multi-method longitudinal twin study of 177 monozygotic and 134 dizygotic twin pairs (i.e., 622 individual infants), including a 5-month lab assessment and multiple follow-up questionnaire-based assessments until 36 months. The study includes EEG, eye-tracking, and genetics, together with more traditional measures based on in-person testing, direct observation and questionnaires based on parent-report. The results show that interest in participation in research among twin families is high, despite the comprehensive protocol. DNA analysis from saliva samples was possible in virtually all participants, allowing for both zygosity confirmation and polygenic score analyses. Combining a longitudinal twin design with advanced methodologies in developmental neuroscience and genomics, BATSS represents a new approach in infancy research, which we hope will have impact across multiple disciplines in the coming years.

\section{The Contribution of Cognitive and Noncognitive Skills to Academic Achievement Throughout the School Years}

Margherita Malanchini ${ }^{1,2}$, Andrea G. Allegrini ${ }^{2,3}$, Michel G. Nivard ${ }^{4}$, Pietro Biroli ${ }^{5}$, Kaili Rimfeld ${ }^{2}$, Rosa Cheesman ${ }^{6}$, Sophie von Stumm ${ }^{7}$, Perline A. D. Demange ${ }^{4}$, Elsje van Bergen $^{4}$, Andrew D. Grotzinger ${ }^{8}$, Laurel A. Raffington ${ }^{8}$, Javier De la Fuente ${ }^{8}$, K. Paige Harden $^{8}$, Elliot M. Tucker-Drob ${ }^{8}$ and Robert Plomin ${ }^{2}$

${ }^{1}$ School of Biological and Chemical Sciences, Queen Mary University of London, UK, ${ }^{2}$ Social, Genetic and Developmental Psychiatry Centre, King's College London, UK, ${ }^{3}$ Department of Psychology, University College London, UK, ${ }^{4}$ Department of Biological Psychology, Vrije Universiteit Amsterdam, Amsterdam, the Netherlands, ${ }^{5}$ Department of Economics, University of Zurich, Switzerland, ${ }^{6}$ PROMENTA Research Center, Department of Psychology, University of Oslo, Oslo, Norway, ${ }^{7}$ Department of Education, University of York, UK and ${ }^{8}$ Department of Psychology, The University of Texas at Austin, TX, USA

Noncognitive skills are important predictors of academic achievement beyond cognitive competencies. However, the developmental role played by genetic and environmental factors in these associations remains largely unexplored. By triangulating multiple methods, we provide a detailed account of how and why cognitive and noncognitive skills are linked to academic performance from age 7 to 16 in the Twins Early Development Study (TEDS). Noncognitive skills predicted variation in achievement beyond cognitive abilities throughout the school years and their contribution increased over development. Multivariate twin analyses showed that genetic factors accounted for a substantial portion of these associations. A new polygenic score (PGS) of noncognitive skills created extending existing approaches to quantifying the genetics of noncognitive skills from DNA (GWAS-by-subtraction), significantly predicted variation in noncognitive traits and academic achievement at every developmental stage, even before the start of formal schooling. While the cognitive PGS prediction remained stable developmentally, the noncognitive PGS prediction nearly doubled from age 7 to 16 , which suggests gene-environment correlation (rGE) is at play. Within-family analyses corroborated this finding and pointed to active rGE. No gene-environment interaction was evident. By studying genetic effects through a developmental lens, we provide new crucial insights into the role of cognitive and noncognitive characteristics in academic development.

\section{'N-of-1 Twin Pair Studies' — An Overview}

Petra J. G. Zwijnenburg

Department of Clinical Genetics, Amsterdam University Medical Center, Amsterdam, the Netherlands

In total, rare diseases (defined as a condition with a prevalence of less than 1:2000) affect 1 in 17 people, millions worldwide. $80 \%$ of these rare conditions are (mono)genetically determined. The increase in the insight in these disorders elucidates therapeutical targets and enables the development of new treatments or provides a rationale of applying therapies used in other conditions. However, small numbers of patients in rare diseases and the lack of evidence or unfeasibility to perform a RCT results in patients missing out on possibly effective interventions. The introduction of so called ' $N$-of-1 studies' (randomized, controlled, multiple crossover trials in a single patient) augments great potential to provide evidence of effectiveness for individuals as well as groups of patients. In unique situations of a concordant monozygotic (MZ) twin pair affected with a rare disease, a clinical in trial in a single twin pair can be very informative and may overcome limitations of ' $N$-of- 1 studies'. An overview will be given of published ' $N$-of-1 Twin Pair studies' and the potential of these studies.

\section{Gene-Environment Interplays Between Family Chaos and Emotional Problems Among Nigerian Adolescents: A Twin Study}

Olakunle A. Oginni ${ }^{1}$ and Yoon-Mi Hur ${ }^{2}$

${ }^{1}$ Department of Mental Health, Obafemi Awolowo University, Ile-Ife, Nigeria and ${ }^{2}$ College of General Education, Kookmin University, Seoul, South Korea

Background: Despite the high child and adolescent mental health burden in low- and middle-income (LMI) settings like Nigeria, little research has investigated gene-environment interplays in mental health risk among adolescents in these settings. We used the classical twin design to investigate gene-environment correlation and interaction between family chaos and emotional problems among Nigerian adolescents. Method: The sample comprised 3207 
Nigerian monozygotic and dizygotic twins aged 12-18 years assessed for emotional problems and family chaos between 2010 and 2012. Family chaos was specified as a moderator of the genetic and environmental influences on emotional problems using a bivariate moderation model. Gene-environment correlations were tested and adjusted for by including the moderator as a dependent variable. Analyses were carried out using structural equation modelling in OpenMx. Results: Family chaos correlated significantly with emotional problems $(r=.30 ; 95 \%$ CI $[0.27,0.34])$, this relationship was significantly influenced by genetic (A $-49 \%$, 95\% CI [0.01, 0.97]) and non-shared environmental factors (E-32\%, 95\% CI $[0.10,0.54])$. Moderation effects were significant whereby as family chaos increased, $\mathrm{A}$ influences on emotional problems decreased $(\beta \mathrm{A}$ $=-0.07,95 \% \mathrm{CI}[-0.12,-0.02])$ while $\mathrm{E}$ influences increased $(\beta \mathrm{E}=0.06,95 \% \mathrm{CI}[0.03,0.09])$. Conclusions: Our findings indicate a gene-environment correlation whereby family chaos may mediate genetic risk for emotional problems among young people. However, the negative moderation found in this study suggests that decreased protective genetic influences on emotional problems may be salient in understanding moderation effects of family chaos in LMI settings. However, these relationships need to be investigated earlier in development.

\section{Same-Sex Attraction and Psychological Distress and Risky Sexual Behaviors: Bidirectional Causal Effects Using the Mendelian Randomization-Direction of Causation Model}

Olakunle A. Oginni ${ }^{1,2}$, Kai Lim ${ }^{1}$, Kirstin L. Purves ${ }^{1}$, Yi Lư ${ }^{3}$, Ada Johansson ${ }^{4}$, Patrik Jern ${ }^{4}$ and Frühling V. Rijsdijk ${ }^{1}$

${ }^{1}$ King's College London, UK, ${ }^{2}$ Obafemi Awolowo University, Ile-Ife, Nigeria, ${ }^{3}$ Karolinska Institutet, Stockholm, Sweden and ${ }^{4}$ Åbo Akademi University, Åbo, Finland

Introduction: Although minority stress explains health disparities among same-sex attracted compared to heterosexual individuals; there is limited evidence to infer causation. This study determined whether same-sex attraction was causally associated with psychological distress and risky sexual behavior. Method: The sample comprised monozygotic and dizygotic twins, and non-twin siblings ( $n=2036,3780$ and 2356 respectively) genotyped and assessed for same-sex attraction, psychological distress, and risky sexual behavior. Causal influences were investigated with same-sex attraction as the predictor and psychological distress and risky sexual behavior as the outcomes in two separate Mendelian Randomization-Direction of Causation (MRDoC) models using OpenMx in R. Supplementary analyses tested reverse causal influences from psychological distress and risky sexual behavior towards same-sex attraction in separate MRDoC models, and moderation of all causal paths by early-life adversities. Results: There were significant causal influences flowing from same-sex attraction to psychological distress and risky sexual behavior (standardized coefficients $=0.13$ and 0.16 ; 95\% CIs $[0.03$, $0.23]$ and $[0.08,0.25]$ respectively). Supplementary analyses further indicated causal influences flowing from psychological distress and risky sexual behavior towards same-sex attraction. Exploratory analyses indicated no significant moderation of causal paths by early-life adversities. Conclusion: Causal influences from same-sex attraction to psychological distress and risky sexual behavior may reflect minority stress which reinforces ongoing measures to minimize social disparities. Causal influences flowing in the opposite direction may reflect rejection sensitivity, stigma-inducing outcomes of risky sexual behavior and recall bias; however, further research is required to specifically investigate these processes.

\section{Heritability of Iris Reflectance Quantified in a Classic Twin Study}

Xiaofan Jiang ${ }^{1,2,4}$, Andrea Kimberly Uy ${ }^{1,2}$, Alex Tanner ${ }^{2}$, Mark Simcoe ${ }^{1,2}$,

Diana Kozareva ${ }^{1,2}$, Katie M. Williams ${ }^{1,2}$, Pirro G. Hysi ${ }^{1,2}$, Christopher J. Hammond ${ }^{1,2}$ and Omar A. Mahroo $1,2,3,4,5$

${ }^{1}$ Department of Twin Research and Genetic Epidemiology, King's College London, St Thomas' Hospital Campus, London, UK, ${ }^{2}$ Institute of Ophthalmology, University College London, UK, ${ }^{3}$ Department of Ophthalmology, King's College London, St Thomas' Hospital Campus, London, UK, ${ }^{4}$ Imperial College School of Medicine, Imperial College, London, UK and ${ }^{5}$ Moorfields Eye Hospital, London

Purpose: To estimate heritability of quantitative iris reflectance measurements and to explore whether these measurements differ significantly between eye color categories. Methods: Video images of eyes of participants from the TwinsUK cohort were obtained from a portable electroretinography device (RETeval, LKC technologies Inc., Gaithersburg, MD, USA) while participants were undergoing electroretinogram recordings. Iris pixel intensity (divided by that recorded from the pupil) was extracted from these images by the device's software. Coefficients of intra-pair correlation for monozygotic (MZ) and dizygotic (DZ) twin pairs were calculated, and heritability was estimated by structural equation modelling. Iris color was also noted (categorized as blue, grey, green, hazel, light brown or dark brown). Measurements were compared between iris color categories (one-way ANOVA). Results: Images were obtained from 1626 participants. After exclusion of singletons, data were available from 1378 twins (456 MZ and $233 \mathrm{DZ}$ pairs); 84\% were female. Mean $(S D)$ age was 52.5 (16.3) years for the whole cohort. Mean $(S D)$ ages were 50.1 (16.6) and 57.1 (14.9) for $\mathrm{MZ}$ and DZ twins respectively. Coefficients of intrapair correlation were 0.75 for MZ pairs and 0.44 for DZ pairs. Age-adjusted heritability was 0.64 (95\% CI [0.60, 0.69]). Of 15 pair-wise comparisons between eye color categories, mean measurements differed significantly $(p<.05)$ for 10 . The following comparisons were not significant: grey-blue; green-blue; green-grey; hazel-green; light brown-hazel. Conclusions: Iris reflectance measurements showed significant heritability and differed significantly between eye color categories. Over $60 \%$ of the variance in these measurements appeared to be attributable to genetic factors.

\section{Heritability of Retinal Rod and Cone-Driven Responses}

Xiaofan Jiang ${ }^{1,2}$, Zihe $\mathrm{Xu}^{1,2}$, Katie M. Williams ${ }^{1,2,3}$, Andrew R. Webster ${ }^{3}$, Christopher J. Hammond ${ }^{1,2}$, Omar A. Mahroo ${ }^{1,2,3}$ and Pirro G. Hysi ${ }^{1,2}$

${ }^{1}$ Department of Twin Research and Genetic Epidemiology, King's College London, St Thomas' Hospital Campus, London UK, ${ }^{2}$ Section of Ophthalmology, King's College London, St Thomas' Hospital Campus, London and ${ }^{3}$ Moorfields Eye Hospital, London UK

Purpose: Retinal neurons generate electrical responses to light stimuli. These can be recorded in vivo in humans as the electroretinogram (ERG). We investigated heritability of rod and cone-driven responses in a twin study. Methods: ERGs were recorded from 208 adult twins in response to white flashes $(0.67,4.0,13$ and 67 photopic $\mathrm{cd} \mathrm{m}-2 \mathrm{~s})$, delivered in the dark (following 20 min dark adaptation) and then in the presence of a dim blue rod-saturating background (1.0 scotopic and 30 photopic $\mathrm{cd} \mathrm{m}-2$ ). The responses in the dark are driven by rods and cones; those in the blue background are driven by cones. Mathematical subtraction of the latter responses from the former yielded an estimated dark-adapted rod system response. Responses were averaged from several flash presentations; ERG parameters were averaged from both eyes. Coefficients of intra-pair correlation were 
calculated for monozygotic (MZ) and dizygotic (DZ) twins, and heritability estimated using structural equation modelling. Results: Mean (SD) age was 62.5 (11.5) years; $93 \%$ were female. Intrapair correlations were higher for $\mathrm{MZ}$ than $\mathrm{DZ}$ pairs for all parameters. Point estimates of heritability of different ERG parameters ranged from 0.26 to 0.82 . The highest heritability was found for the b-wave amplitude of the cone-driven response to the brightest flash. Conclusion: Our findings indicate that a significant proportion of the variance in both rod and cone-driven responses are attributable to genetic factors. The highest heritability was found in a parameter relating to cone-driven ONbipolar cell responses.

\section{Narrowing in on What Really Matters: Testing Criminological Theory Using Twin-Based Methodology}

Joseph L. Nedelec

School of Criminal Justice, University of Cincinnati, OH, USA

Criminological explanations of antisocial behaviors have typically centered on sociological factors. However, in recent decades scholars taking a biosocial approach have illustrated that virtually every behavioral and systems-based outcome of interest in criminology is partially impacted by variance in genetic factors. Termed biosocial criminology (aka, biopsychosocial criminology), the approach has relied heavily on twin-based methodologies. The bulk of the research in this area of criminology has focused on assessing key hypotheses derived from criminological theory. Relatively absent from this literature, however, is an integration of fundamental components of the nature of twin relationships. For example, sibling relationship quality can differentially impact various behavioral outcomes and this impact appears to vary across different types of twins. Further, twin studies have highlighted that as genetic relatedness increases so too does the overall importance of the quality of the sibling relationship. The long-term influence of sibling relationship quality on criminological outcomes, however, is relatively unknown. With this information as a backdrop, the current presentation reviews some examples of twin-based biosocial criminology and provides a test of the extent to which sibling relationship quality served as an influential factor for delinquency across the life course. Using varying non-twin and twinbased sibships drawn from the National Longitudinal Study of Adolescent to Adult Health, the results illustrate minimal long-term impact of sibling relationship quality on antisocial behavior. However, the pattern of the findings varied across sibling types. Overall, the study illustrates the value of the integration of twinbased methodologies in assessing criminological theory.

\section{Peri/Postnatal Epigenetic Twins Study (PETS) Cohort: Double-One Years}

Jane Loke $^{1,2}$, Richard Saffery ${ }^{1,2}$ and Jeffrey Craig $^{1,2,3}$

${ }^{1}$ Murdoch Children's Research Institute, Melbourne, VIC, Australia, ${ }^{2}$ University of Melbourne, Melbourne, VIC, Australia and ${ }^{3}$ Deakin University, Geelong, VIC, Australia

The PETS cohort was established by Prof. Jeffrey Craig and Prof. Richard Saffery, with the primary aim to investigate the role of early life epigenetics in human development. Epigenetics can be defined as a reversible mechanism that modify gene expression that are not due to changes in DNA sequence. PETS started recruitment in 2006, and since then, we have managed to collect a wide range of pre-/postnatal epidemiological data and biological samples at birth, 18 months, 6 years- and 11-years-old timepoints. With the focus on dental health and neurodevelopmental outcomes at 6 years and 11 years old respective data collection, usage of PETS longitudinal data can address the knowledge gap regarding rate of epigenetic drift during early development, influence of early life environment on later health outcomes mediated by epigenetics, and the interaction of environment and genetics influence on epigenetics. For this presentation, I will present a summary of some published findings from PETS investigators in addition to a brief background of the cohort.

\section{Strengthening Evidence-Based Multiple Birth Family Nursing Care: Literature Reviews}

Kristiina Heinonen ${ }^{1,2}$

${ }^{1}$ Metropolia University of Applied Sciences, Health Promotion, Metropolia, Helsinki, Finland and ${ }^{2}$ University of Eastern Finland, Department of Nursing Science, Kuopio, Finland

Introduction: Multiple birth family is a family with special needs. Research before show that parents would like to have more specific information and attention by social and health care professionals. Methods: A descriptive literature review and systematic knowledge search used by method concentrating to twins/triplets' individuality, sleeping in multiple birth family, and supporting multiple birth parents. The material consisted of reviewed research articles $(n=26)$ and analyzed by inductive content analysis. Results: The result showed that there are many evidence-based research results which can be used by social and health care professionals. The results highlighted the importance of support and consideration for individuality. Twins' relationship has a significant impact on the individual, health, and wellbeing. The results also show that multiple birth family parents suffer from sleeping deprivation and use different kind of methods to cope and promote to sleeping in a family. To support for the family and close family members, peer support has become an important part of the need for support. Discussing with parents and giving guidance by social and health care professionals promote the health and well-being in the whole family. Conclusions: More research and education needed to evidence-based family nursing care in the different context. The social- and health care professionals need further tools and training to be able to empower parents of twins. It is also important to support social and health care students' possibility to do multiple birth family research/thesis.

\section{Disentangling the Impact of Genetics and Environment on Brain Functional Connections: A 3T Resting State fMRI Study on Young Twins}

Emma Tassi ${ }^{1}$, Eleonora Maggioni ${ }^{2}$, Maddalena Mauri ${ }^{3}$, Corrado Fagnani ${ }^{4}$, Filippo S. Arrigoni ${ }^{5}$, Anna M. Bianchi ${ }^{1}$, Maria A. Stazi ${ }^{4}$, Maria Nobile ${ }^{3}$ and Paolo Brambilla ${ }^{2,6}$

${ }^{1}$ Department of Electronics, Information and Bioengineering, Politecnico di Milano, Milan, Italy, ${ }^{2}$ Department of Neurosciences and Mental Health, Fondazione IRCCS Ca' Granda Ospedale Maggiore Policlinico, Milan, Italy, ${ }^{3}$ Child Psychopathology Unit, Scientific Institute, IRCCS Eugenio Medea, Bosisio Parini (LC), Italy, ${ }^{4}$ Centre for Behavioural Sciences and Mental Health, Istituto Superiore di Sanità, Rome, Italy, ${ }^{5}$ Neuroimaging Lab, Scientific Institute, IRCCS E. Medea, Bosisio Parini, Italy and ${ }^{6}$ Department of Pathophysiology and Transplantation, University of Milan, Milan, Italy

Introduction: Resting-state network (RSN) interindividual variability that arises during developmental age is driven by a complex interplay between genetics and environment. Twin studies provide an optimal platform for the analysis of genetic and environmental influences on specific RSN characteristics. In this study, we applied statistical twin methods to gain quantitative insight into the determinants of intrinsic 
functional connectivity among brain regions. Method: A 3T restingstate functional magnetic resonance imaging ( $r$-fMRI) dataset from 43 same-sex twin pairs, 25 dizygotic (DZ) and 18 monozygotic (MZ), was employed for the analyses. Functional connectivity (FC) measures between brain regions of interest (ROI) were extracted. $\mathrm{MZ}$ and DZ intra-pair correlations were compared to assess the twin design applicability. When possible, genetic and environmental contributions to link-level FC features were jointly estimated by fitting ACE or ADE twin models, as appropriate. Genetic influences at multiple loci, that is, epistatic effects, were also assessed. Results: The $9.61 \%$ of functional connections met ACE model criteria, with prominent shared environmental effects in right fusiform links. The $7.97 \%$ of FC links - many involving orbitofrontal and middle temporal pole - showed epistatic effects. The $7.14 \%$ of functional connections involving the middle temporal pole as well — were found to be influenced by ADE factors. Conclusions: Our preliminary findings can provide considerable insights into the uncharted genetic and environmental influences on functional brain networks during the developmental period. Our evidence suggests genetic control over frontotemporal connections, but also underlines a potential role of shared environment in shaping temporooccipital connections.

\section{The Neural Bases of Social Cognitive Interference in Young Twins Discordant for Psychotic Risk}

Cinzia Molent ${ }^{1,2}$, Cinzia Molent ${ }^{1,2}$, Eleonora Maggioni ${ }^{3}$, Carolina Bonivento ${ }^{4}$, Vaibhav Diwadkar ${ }^{5}$, Maddalena Mauri ${ }^{6}$, Corrado Fagnani ${ }^{7}$, Maria A. Stazi ${ }^{7}$, Maria Nobile ${ }^{6}$ and Paolo Brambilla ${ }^{3,8}$

${ }^{1}$ Genetics of Cognition Laboratory, Neuroscience Area, Istituto Italiano di Tecnologia, Genova, Italy, ${ }^{2}$ Dipartimento di Medicina Sperimentale, (Di. Mes) Università degli Studi di Genova, Genova, Italy, ${ }^{3}$ Department of Neurosciences and Mental Health, Fondazione IRCCS Ca' Granda Ospedale Maggiore Policlinico, Milan, Italy, ${ }^{4}$ Unit of Psychiatry, Department of Medicine (DAME), University of Udine, Udine, Italy, ${ }^{5}$ Department of Psychiatry \& Behavioral Neuroscience, Wayne State University, Detroit, MI, USA, ${ }^{6}$ Child

Psychopathology Unit, Scientific Institute, IRCCS Eugenio Medea, Bosisio Parini (LC), Italy, ${ }^{7}$ Centre for Behavioural Sciences and Mental Health, Istituto Superiore di Sanità, Rome, Italy and ${ }^{8}$ Department of Pathophysiology and Transplantation, University of Milan, Milan, Italy

Introduction: Social cognition deficits have been described in psychotic disorders and high-risk psychotic states (HRP). Understanding the brain correlates of such impairments in HRP subjects and their link with genetics end environment is needed to develop early recognition and intervention strategies in psychosis. Methods: Thirteen young twin pairs (8 monozygotic, 5 dizygotic, $17.4 \pm 5.2$ years) discordant for psychotic risk based on the ERIRaos-CheckList and 13 age- and sex-matched controls (HC) underwent a fMRI session during an emotional continuous performance test. The test was designed to assess cognitive-emotional interference by combining Go/NoGo with facial emotion recognition. The fMRI activations were compared between HRP and their low-risk twins (LRP) and HC via paired and independent t-tests, respectively. Results: During recognition of negative faces vs. nonfaces, HRP had greater activation than $\mathrm{HC}$ in hubs of the default mode network and in the postcentral cortex, suggesting their possible role in the abnormal negative emotion interference characterizing psychotic risk. During recognition of neutral faces vs. non-faces, HRP showed greater activation than (i) all other groups in temporal and precentral cortices, (ii) their LRP twins in supramarginal and lingual cortices. Thus, higher recruitment of these regions seems required for cognitive control over social information in HRP but not in their LRP twins. Conclusions: Specific activations in brain regions involved in cognitive control over socially-relevant stimuli seem to differentiate HRP from their unaffected twins, indicating that factors other than heritability, intrauterine and familiar environment could play a role in psychosis vulnerability.

\section{Genetic Impacts on DNA Methylation Help Dissect the Interplay Between Genetics, Epigenetics and Gene Expression}

Sergio Villicaña ${ }^{1}$, Juan Castillo-Fernandez ${ }^{1}$, Eilis Hannon ${ }^{2}$, Colette Christiansen ${ }^{1}$, Pei-Chien Tsai ${ }^{1}$, Jane Maddock ${ }^{3}$, Diana Kuh ${ }^{3}$, Matthew Suderman ${ }^{4}$, Christine Power ${ }^{5}$ Caroline Relton ${ }^{4,5}$, George Ploubidis ${ }^{6}$, Andrew Wong ${ }^{3}$, Rebecca Hardy ${ }^{7}$,

Alissa Goodman ${ }^{6}$, Ken Ong ${ }^{8}$ and Jordana T. Bell ${ }^{1}$

${ }^{1}$ Department of Twin Research and Genetic Epidemiology, King's College London, UK, ${ }^{2}$ University of Exeter Medical School, Exeter, UK, ${ }^{3} \mathrm{MRC}$ Unit for Lifelong Health and Ageing, Institute of Cardiovascular Science, University College London, UK, ${ }^{4}$ MRC Integrative Epidemiology Unit, University of Bristol, Bristol, UK, ${ }^{5}$ Population, Policy and Practice Research and Teaching Department, UCL Great Ormond Street Institute of Child Health, London, UK, ${ }^{6}$ Centre for Longitudinal Studies, Institute of Education, University College London, UK, ${ }^{7}$ CLOSER, UCL Social Research Institute, University College London, UK and ${ }^{8} \mathrm{MRC}$ Epidemiology Unit and Department of Paediatrics, Wellcome Trust-MRC Institute of Metabolic Science, University of Cambridge School of Clinical Medicine, Cambridge, UK

Background: DNA methylation (DNAm) levels at a proportion of sites across the genome are influenced by genetic variants, or methylation quantitative trait loci (meQTLs). The primary purpose of this work was to identify meQTLs through genome-wide association analyses and to better understand the relationship between DNA methylation and gene expression. Methods: Blood DNA samples were obtained from 2,358 participants from three UK-based population cohorts including TwinsUK, the MRC National Survey for Health and Development or 1946 birth cohort, and the National Child Development Study or 1958 birth cohort. We applied a classical twin study to estimate genome-wide heritability of DNAm at $\sim 720 \mathrm{k}$ sites profiled by the Illumina Infinium MethylationEPIC BeadChip. A following association analyses compared genotypes and DNAm levels. We then performed Summary-based Mendelian Randomization (SMR) and tested pleiotropic associations between DNAm and gene expression levels for 19,250 genes. Results: We estimated a mean DNAm heritability of 0.14 . Methylation levels at $34 \%$ of the DNAm sites genome-wide were affected by meQTLs. MeQTL SNPs and associated DNAm sites tend to be located in specific genomic regions (e.g., enhancers and insulators). After SMR analyses we found that meQTL SNPs colocalize with putative causal variants for gene expression of $44 \%$ of tested genes. The results provide suggestive evidence of specific examples of hypothesized underlying mechanisms of genetic regulation on DNAm. Conclusion: The results improve the characterization of genetic influences on DNA methylation and are informative towards the prioritization of genetic variants for functional follow-up analysis in genomewide association studies.

\section{Gene-by-Crisis Interaction for Optimism and Meaning in Life: The Effects of the COVID-19 Pandemic}

Lianne P. de Vries ${ }^{1,2}$, Margot P. van de Weijer ${ }^{1,2}$, Dirk H. M. Pelt ${ }^{1,2}$, Lannie Ligthart ${ }^{1,2}$, Gonneke Willemsen ${ }^{1,2}$, Dorret I. Boomsma ${ }^{1,2}$, Eco J. C. de Geus ${ }^{1,2}$ and Meike Bartels ${ }^{1,2}$

${ }^{1}$ Department of Biological Psychology, Vrije Universiteit Amsterdam, Amsterdam, the Netherlands and ${ }^{2}$ Amsterdam Public Health Research Institute, Amsterdam University Medical Centres, Amsterdam, The Netherlands

The corona virus disease 2019 (COVID-19) pandemic and the restrictions to reduce the spread of the virus has had a large impact 
on daily life. We investigated the individual differences in the effect of the COVID-19 pandemic and first lockdown on optimism and meaning in life in a sample from the Netherlands Twin Register. Participants completed surveys before $(N=9964)$ and during the pandemic (i.e., April-May 2020, $N=17,464$ ), with a subsample completing both surveys $(\mathrm{N}=6461)$. We applied longitudinal twin models to investigate changes in the genetic architecture of the traits due to the pandemic and the gene-environment interaction. Although $56 \%$ and $35 \%$ of the sample was negatively affected by the pandemic, many participants were stable $(32 \%$ and $43 \%)$ or even showed increased optimism and meaning in life (11\% and 22\%). Subgroups, specifically women and people with poorer health, experienced larger negative effects. During the pandemic, slightly lower heritability estimates for optimism (20\%) and meaning in life (25\%) were obtained compared to prepandemic (respectively $26 \%$ and $32 \%)$, although confidence intervals overlap. The lower than unity genetic correlations (.75 and .63) suggest gene-environment interactions, where the expression of genes that influence optimism and meaning in life differs. The COVID-19 pandemic is a strong exposure leading to imbalanced effects on the well-being of individuals. The differences are partly explained by individual differences in genetic sensitivity to environmental change. More knowledge on the person-specific response to specific environmental variables underlying these individual differences is needed to prevent inequality.

\section{The Multiple Birth Cohort Study: A Birth Cohort within the Italian Twin Registry}

Sonia Brescianini

Center for Behavioral Sciences and Mental Health, Istituto Superiore di Sanità, Rome, Italy

The Italian Twin Registry (ITR), established in 2001, is a populationbased registry of voluntary twins. To date, it consists of approximately 29,000 twins of all ages who gave their consent to participate in the studies proposed by the ITR research group. The Multiple Births Cohort Study (MUBICOS) is a cohort of newborn twins, within the framework of the ITR, which enrolled newborn twins in 8 different hospitals. Hospitals are spread around the whole country, 3 in the north, 3 in the center and 2 in the south of Italy. The cohort started in 2010 has now enrolled about 360 twin pairs and their parents. Follow-up questionnaires were sent at $6,12,18$ and 36 months and a new follow up is planned around 10-12 years of age. One of the unique characteristics of the cohort, among birth cohorts, is that we collected DNA from saliva from both twins and both parents establishing one of the few family collections of DNA with connected health information. The DNA is stored in the ITR Biobank and a genomewide genotyping has been performed. Since the establishment of the cohort, the twins underwent a focus on respiratory health and its relationship with growth in the first year of life. In detail, the association between growth in infancy and wheezing at 3 years of age is decomposed into genetic and environmental components using twin modeling techniques based on estimating between- and within-pair correlations. Furthermore, MUBICOS participates in different birth cohort international networks.

\section{Phenotypic Associations and Health Outcomes of Age-Acquired Skewed X Chromosome Inactivation in Humans}

Amy L. Roberts ${ }^{1}$, Alessandro Morea ${ }^{1,2,3}$, Ariella Amar ${ }^{2}$, Antonino Zito ${ }^{1,4}$, Julia S. El-Sayed Moustafa $^{1}$, Max Tomlinson ${ }^{1,2}$, Ruth Bowyer ${ }^{1}$, Xinyuan Zhang ${ }^{1}$, Colette Christiansen ${ }^{1}$, Ricardo Costeira ${ }^{1}$, Claire J. Steves ${ }^{1}$, Massimo Mangino ${ }^{1}$, Jordana T. Bell ${ }^{1}$, Chloe C. Y. Wong ${ }^{5}$, Timothy J. Vyse ${ }^{2}$ and Kerrin S. Small ${ }^{1}$

${ }^{1}$ Department of Twin Research \& Genetic Epidemiology, King's College London, London, UK, ${ }^{2}$ Department of Medical and Molecular Genetics, King's College London, London, UK, ${ }^{3}$ The FIRC Institute of Molecular Oncology, Italian Foundation for Cancer Research, Milan, Italy, ${ }^{4}$ Department of Molecular Biology, Massachusetts General Hospital, Boston, USA; Department of Genetics, Harvard Medical School, Boston, MA, USA and ${ }^{5}$ Social, Genetic \& Developmental Psychiatry Centre, Institute of Psychiatry, Psychology \& Neuroscience, King's College London, London, UK

Aging is a heterogenous process characterised by cellular and molecular hallmarks and is a primary risk factor for chronic diseases. $\mathrm{X}$ chromosome inactivation (XCI) randomly transcriptionally silences either the maternal or paternal $\mathrm{X}$ in each cell of XX, 46 females to equalise the gene expression with XY,46 males. However, some individuals display preferential inactivation of one of the two $\mathrm{X}$ chromosomes, and this imbalance can increase across the lifecourse. Age acquired skewed X-inactivation is particularly prevalent in blood tissues of aging females and is thought to be due to changes to the haematopoietic stem cell population. We used the HUMARA assay to measure XCI in 1575 females from TwinsUK with date-matched complete blood count data and health records. Associations with atherosclerosis and cardiovascular disease (ASCVD) risk and blood count data were tested using linear mixed effects models. A prospective 10-year follow-up study of cancer diagnoses was analyzed using Cox multivariate regression. XCI-skew is independent of traditional markers of biological aging, including epigenetic aging, telomere length shortening, and frailty. XCI-skew is associated with increased monocyte abundance $(p=.004)$, a cell type known to be implicated in cardiovascular disease, and general increases in cells of the myeloid lineage. XCI-skew is associated with an increase of ASCVD risk both cross-sectionally $(p=.01)$ and within discordant twin pairs $(p=.025)$, and increased risk of cancer $(p=.032$; hazard ratio $(\mathrm{HR})=1.74(95 \% \mathrm{CI}[1.05,2.89])$. In summary, age acquired $\mathrm{XCI}-$ skew is associated with increased risk of chronic diseases, potentially driven by changes to the haematopoietic stem cell population.

\section{Changes in Physical Activity Levels and Mental Health During COVID-19: Prospective Findings From Studying Adult Twin Pairs}

John Hopper ${ }^{1}$, Glen E. Duncan ${ }^{2}$, Ally R. Avery ${ }^{2}$, Siny Tsang ${ }^{2}$, Bethany D. Williams ${ }^{2}$ and Edmund Seto ${ }^{3}$

${ }^{1}$ Melbourne School of Population and Global Health, The University of Melbourne, VIC, Australia, ${ }^{2}$ Department of Nutrition and Exercise Physiology, Washington State University, WA, USA and ${ }^{3}$ University of Washington, Seattle, WA, USA

Background: The purpose of this study was to investigate prospectively associations between changes in physical activity (PA) and mental health outcomes during the COVID-19 pandemic, following 
implementation of mitigation strategies, using a sample of adult twins. Methods: This was a prospective study of 3,057 adult twins from the Washington State Twin Registry who had completed online surveys at baseline (March 26 - April 5, 2020), and three follow-up waves (W1: April 20 - May 3; W2: July 16 - August 2; W3: September 16 - October 1). PA was operationalized as self-reported moderate-tovigorous PA (MVPA) and neighborhood walking (minutes/week). Mental health outcomes were operationalized as self-reported anxiety and perceived stress as measured at follow-up. Latent growth curve models (LGCMs) were used to assess changes in PA and mental health outcomes over time, estimating the cross-sectional, parallel, and prospective associations between PA and mental health over time while taking into account within-pair correlations and adjusted for age, sex, and race. Results: MVPA and walking decreased over time, levels of anxiety remained stable, and stress increased slightly. Cross-sectional associations between both PA predictors and mental health outcomes were weak at baseline. After taking into account cross-sectional associations between PA and mental health outcomes, changes in PA over time were not associated with changes in mental health outcomes over time. Conclusions: Over a time period aligned with COVID-19 mitigation strategies and social restrictions, changes in physical activity was not detectably associated with changes in anxiety or stress levels. Nonetheless, the average decline in PA over time is worrisome. Public health resources should continue to promote PA as a means to improve physical health during the pandemic.

\section{Perceived Change in Physical Activity Levels and Mental Health During COVID-19: Findings Among Adult Twin Pairs}

Catherine Bennett ${ }^{1}$, Glen E. Duncan ${ }^{1}$, Ally R. Avery ${ }^{1}$, Edmund Seto ${ }^{2}$ and Siny Tsang ${ }^{1}$

${ }^{1}$ Department of Nutrition and Exercise Physiology, Washington State

University, WA, USA and ${ }^{2}$ University of Washington, Seattle, WA, USA

Background: Physical distancing and other COVID-19 pandemic mitigation strategies may have unintended consequences on a number of health behaviors and health outcomes. The purpose of this study was to investigate associations between perceived change in physical activity or exercise and mental health outcomes over the short-term in response to COVID-19 mitigation strategies in a sample of adult twins. Methods: This was a cross-sectional study of 3971 identical and same-sex fraternal adult twins (909 pairs, 77\% identical) from the community-based Washington State Twin Registry. Participants completed an online survey examining the impact of COVID-19 mitigation on a number of health-related behaviors and outcomes, administered between March 26 and April 5, 2020. Exposure was perceived change in physical activity or exercise and outcomes were levels of perceived anxiety and stress. Results: More twin pairs reported a decrease in physical activity levels (42\%) than no change $(31 \%)$ or increased levels $(27 \%)$. A perceived decrease in physical activity or exercise was associated with higher stress and anxiety levels. However, the physical activity-stress relationship was confounded by genetic and shared environmental factors. On the other hand, the physical activity - anxiety relationship held after controlling for genetic and shared environmental factors, although no longer significant after controlling for age and sex, with older twins more likely to report lower levels of anxiety and females more likely to report higher. Conclusions: Strategies to mitigate the
COVID-19 pandemic may be impacting physical activity and mental health, with those experiencing a decrease in physical activity also having higher levels of stress and anxiety. These relationships are confounded by genetic and shared environmental factors in the case of stress, and age and sex in the case of anxiety.

\section{Global Applied Learning Experience Project, A Mixed Method Study Protocol}

Shayesteh Jahanfar ${ }^{1}$, , Lynch $^{2}$, Pooja Patel ${ }^{2}$ and Mojgan Gharipour ${ }^{2}$

${ }^{1}$ Tufts School of Medicine, Department of Public Health and Community Medicine, Boston, MA, USA and ${ }^{2}$ Division of Genetics and Epigenetics Studies, Cardiovascular Research Institute, Isfahan University of Medical Sciences, Isfahan, Iran

Introduction: Twin studies are unique. Aim: The objective is to obtain stakeholders' attitudes, beliefs, desires, and experiences in establishing a twin registry, and to obtain risk factors associated with anxiety/depression among twins. Methodology: This is a mixed method study of twins registered at Isfahan Twin Registry (ITR). As a part of Global Applied Learning Experience at Tufts, we reached out to ITR to establish a research-training activity, aiming to gain skill sets required by $\mathrm{CEPH}$ accreditation for the MPH program. This project will begin with a literature review of twin studies. We intend to attend the 19th International Twin Conference to enrich our understanding of twin studies. Equipped with the background information of how twin registries are established across the globe, we will design a list of structured questions to conduct the qualitative part of our study. We will then communicate with the management team at ITR and with the identify individuals responsible for establishing twin registries at global level to interview 20 stakeholders about challenges and feasibility of creating a population-based national twin registry. For the quantitative part of our study, we will work on collected data of adults at the ITR. We will conduct a secondary data analysis using cluster analysis to ensure twins correlation is taken into account. Results: We will build two predictive regression models, one for each specific objective. We will then prepare knowledge translation pieces to share with scientific communities as well as the public.

\section{Identical Twins Carry a Persistent Epigenetic Signature of Early Genome Programming}

Jenny van Dongen ${ }^{1,2,3}$, Scott D. Gordon ${ }^{4}$, Allan F. McRae ${ }^{5}$, Veronika V. Odintsova ${ }^{1,2,3}$, Hamdi Mbarek ${ }^{1,2,3}$, Charles E. Breeze ${ }^{6}$, Karen Sugden ${ }^{7}$, Sara Lundgren ${ }^{8}$, Juan E. CastilloFernandez ${ }^{9}$, Eilis Hannon ${ }^{10}$, Terrie E. Moffitt ${ }^{7,11}$, Fiona A. Hagenbeek ${ }^{1,3}$, Catharina E. M. van Beijsterveldt ${ }^{1,3}$, Jouke Jan Hottenga ${ }^{1,3}$, Pei-Chien Tsai ${ }^{9}$ BIOS Consortium ${ }^{\star}$ The Genetics of DNA Methylation Consortium ${ }^{\star \star}$, Josine L. Min ${ }^{12,13}$, Gibran Hemani ${ }^{12,13}$, Erik A. Ehli ${ }^{14}$, Franziska Paul ${ }^{15}$, Claudio D. Stern ${ }^{16}$, Bastiaan T. Heijmans ${ }^{17}$, P. Eline Slagboom ${ }^{17}$ Lucia Daxinger ${ }^{18}$, Silvère M. van der Maarel ${ }^{18}$, Eco J.C. de Geus ${ }^{1,3}$, Gonneke Willemsen ${ }^{1,3}$, Grant W. Montgomery ${ }^{5}$, Bruno Reversade ${ }^{15,19,20}$, Miina Ollikainen ${ }^{8}$, Jaakko Kaprio ${ }^{8}$, Tim D. Spector ${ }^{9}$, Jordana T. Bell ${ }^{9}$, Jonathan Mill ${ }^{10}$, Avshalom Caspi ${ }^{7,11}$, Nicholas G. Martin ${ }^{4}$ and Dorret I. Boomsma ${ }^{1,2,3}$

${ }^{1}$ Department of Biological Psychology, Vrije Universiteit Amsterdam, Amsterdam, the Netherlands, ${ }^{2}$ Amsterdam Reproduction and Development (AR\&D) Research Institute, Amsterdam, the Netherlands, ${ }^{3}$ Amsterdam Public Health Research Institute, Amsterdam, the Netherlands, ${ }^{4}$ QIMR Berghofer Medical Research Institute, Brisbane, QLD, Australia, ${ }^{5}$ Institute for Molecular Bioscience, The University of Queensland, Brisbane, QLD, Australia, ${ }^{6}$ Altius Institute for Biomedical Sciences, Seattle, WA, USA, ${ }^{7}$ Department of Psychology and Neuroscience and Center for Genomic and Computational Biology, Duke University, Durham, NC, USA, ${ }^{8}$ Institute for Molecular Medicine Finland FIMM, University of Helsinki, Helsinki, Finland, ${ }^{9}$ Department of Twin Research and Genetic Epidemiology, Kings College London, London, UK, 


\begin{abstract}
${ }^{10}$ University of Exeter Medical School, University of Exeter, Exeter, UK, ${ }^{11}$ Institute of Psychology, Psychiatry \& Neuroscience, King's College London, UK, ${ }^{12} \mathrm{MRC}$ Integrative Epidemiology Unit at the University of Bristol, Bristol, UK, ${ }^{13}$ Population Health Science, Bristol Medical School, University of Bristol, Bristol, UK, ${ }^{14}$ Avera Institute for Human Genetics, Sioux Falls, SD, USA, ${ }^{15}$ Institute of Molecular and Cellular Biology, A*STAR, Singapore,

${ }^{16}$ Department of Cell and Developmental Biology, University College London, London, UK, ${ }^{17}$ Molecular Epidemiology, Department of Biomedical Data Sciences, Leiden University Medical Center, Leiden, the Netherlands, ${ }^{18}$ Department of Human Genetics, Leiden University Medical Center, the Netherlands, ${ }^{19} \mathrm{Genome}$ Institute of Singapore, A*STAR, Singapore and ${ }^{20}$ Medical Genetics Department, KOC University, School of Medicine, Istanbul, Turkey
\end{abstract}

Monozygotic (MZ) twins and higher-order multiples arise when a zygote splits during pre-implantation stages of development. The mechanisms underpinning this event have remained a mystery. Because MZ twinning rarely runs in families, the leading hypothesis is that it occurs at random. Here, we show that $\mathrm{MZ}$ twinning is strongly associated with a stable DNA methylation signature in adult somatic tissues. This signature spans regions near telomeres and centromeres, Polycomb-repressed regions and heterochromatin, genes involved in cell-adhesion, WNT signaling, cell fate, and putative human metastable epialleles. Our study also demonstrates a never-anticipated corollary: because identical twins keep a lifelong molecular signature, we can retrospectively diagnose if a person was conceived as monozygotic twin.

\section{Biology of Cancer Survivorship in Twins with Hodgkin Lymphoma}

Wendy Cozen ${ }^{1}$, Marta Epeldegui ${ }^{2}$, Amie Eunah Hwang ${ }^{3}$, David V. Conti ${ }^{3}$, Jun Wang ${ }^{3}$, Larry Magpantay ${ }^{2}$, Thomas M. Mack ${ }^{3}$, Otoniel Martinez-Maza ${ }^{2}$ and Steve Cole ${ }^{2}$

${ }^{1}$ University of California Irvine, Orange, California, USA, ${ }^{2}$ David Geffen School of Medicine at UCLA, Los Angeles, California, USA and ${ }^{3}$ Keck School of Medicine at the University of Southern California, Los Angeles, California, USA

Background: Hodgkin lymphoma (HL) occurs primarily in adolescents and young adults (AYA) in developed countries and is associated with immune dysregulation. We found that AYAHL survivors had accelerated DNA methylation age, altered gut microbiota, higher EBV viral load and levels of circulating immune biomarkers compared to their unaffected co-twins. To identify differences in immune gene transcription control pathways, we examined mRNA profiles from 17 pairs of long term AYAHL survivors and their unaffected twins. Methods: Blood samples were collected remotely, and B and $\mathrm{T}$ cells were negatively separated. RNA was extracted and sequenced using a high-efficiency mRNA-targeted assay system (average 6.7 million reads per sample) mapped to the hg38 human transcriptome (99\%). Raw read counts were normalized to transcripts per million total mapped reads and $\log 2$ transformed for analysis using linear statistical models including a random effect of twin pair. Results: T-cells of survivors showed marginally lower expression of 12 activation-induced genes $(0.41$-fold, $p=.038)$ and markedly lower expression of 28 Type 1 interferon-related genes (0.33-fold, $p<.001)$ relative to their unaffected co-twins. B cells showed similar reductions in activation-induced genes $(0.33$-fold, $p<.001)$ but no difference in Type 1 interferon genes (1.02-fold, $p=.942)$. We also identified marked up-regulation of multiple GATA family transcription factors (GATA3: $p<.001$; GATA1: $p=.009$; GATA2: $p=.032)$. Conclusions: These differences further support the premise that abnormalities in T-cells play a strong role in etiology, pathogenesis, and survivorship and how twins can provide an unbiased assessment.

\section{Twin Registers Across Different Cultures}

Yoon-Mi Hur ${ }^{1}$ and Veronika Odintsova ${ }^{2}$

${ }^{1}$ Kookmin University, Seoul, South Korea and ${ }^{2}$ Vrije University, Amsterdam, the Netherlands

Twin registers have contributed to our knowledge about genetic and environmental influences on all aspects of human life. This paper provides a history of twin registers and an overview of twin registers across globe. Twin registers started in Europe, and then, spread to North America, Australia, Asia, increased awareness of the value of twin studies. Currently, about 60 to 70 twin registers actively engage in twin research in 28 developing and developed countries in the world. Early twin registers mostly recruited same-sex twins, but nowadays, many registers recruit family members including siblings, parents, spouses and children of twins as well as opposite-sex twin pairs. Currently, 1.3 to 1.5 million twins and their families are participating in twin research worldwide. Many twin registers are national ones, collecting data by linking their twins to national demographic, education, social, and health registers, which improves the quality of data and ensures representativeness of twin samples. Many registers also collect phenotypic, genetic, epigenetic and microbial information from their participants, which offers great opportunities to study origins of cross-cultural differences and similarities.

\section{Perceived Change in Physical Activity Levels and Mental Health During COVID-19: Findings Among Adult Twin Pairs}

Glen E. Duncan ${ }^{1}$, Ally R. Avery ${ }^{1}$, Edmund Seto ${ }^{2}$ and Siny Tsang ${ }^{1}$

${ }^{1}$ Washington State University, WA, USA and ${ }^{2}$ University of Washington, Seattle, WA, USA

Background: Physical distancing and other COVID-19 pandemic mitigation strategies may have unintended consequences on health behaviors and health outcomes. Our aim was to investigate associations between perceived change in physical activity or exercise and mental health outcomes over the short-term in response to COVID-19 mitigation strategies in a sample of adult twins. Methods: A cross-sectional study of 3,971 identical and same-sex fraternal adult twins (909 pairs, 77\% identical) from the communitybased Washington State Twin Registry. Participants completed an online survey on the impact of COVID-19 mitigation on a number of health-related behaviors and outcomes, administered between March 26 and April 5, 2020. Exposure was perceived change in physical activity or exercise and outcomes were levels of perceived anxiety and stress. Results: More twin pairs reported a decrease in physical activity levels (42\%) than no change (31\%) or an increase (27\%). A perceived decrease in physical activity or exercise was associated with higher stress and anxiety levels. However, the physical activity-stress relationship was confounded by genetic and shared environmental factors. On the other hand, the physical activityanxiety relationship held after controlling for genetic and shared environmental factors, although no longer significant after controlling for age and sex, with older twins more likely to report lower levels of anxiety and females more likely to report higher. Conclusions: 
Strategies to mitigate the COVID-19 pandemic may be impacting physical activity and mental health with those experiencing a decrease in physical activity also having higher levels of stress and anxiety. These relationships are confounded by genetic and shared environmental factors in the case of stress, and age and sex in the case of anxiety.

\section{Genetic and Environmental Influences on Quality of Life and Self-Rated Health: The COVID-19 Pandemic as a Natural Experiment}

Margot P. van de Weijer ${ }^{1,2}$, Lianne P. de Vries ${ }^{1,2}$, Dirk Pelt ${ }^{1,2}$, Floris Huider ${ }^{1,2}$, Matthijs D. van der Zee ${ }^{1,2}$, Lannie Ligthart ${ }^{1,2}$, Gonneke Willemsen ${ }^{1,2}$, Dorret I. Boomsma ${ }^{1,2}$, Eco de Geus ${ }^{1,2}$ and Meike Bartels ${ }^{1,2}$

${ }^{1}$ Department of Biological Psychology, Vrije Universiteit Amsterdam, the Netherlands and ${ }^{2}$ Amsterdam Public Health Research Institute, Amsterdam, the Netherlands

Introduction: The COVID-19 pandemic and consequent lockdowns have impacted both those who were infected and those who remain(ed) uninfected. Given the large impact of lockdowns on daily life and social interactions, an important question is how the pandemic has affected people's (mental) health. Methods: We study the impact of the COVID-19 lockdown in the Netherlands on Self-Rated Health (SRH) and Quality of Life (QoL) using data from uninfected individuals from the Netherlands Twin Register. We compare the variance decomposition before the pandemic to during the pandemic using: (1) bivariate longitudinal twin models for SRH, and 2) pedigree-based analyses in Mendel for QoL. Results: When comparing QoL and SRH levels before the pandemic to during the pandemic, we see that SRH remained stable for most individuals, while QoL mainly decreased. The bivariate longitudinal genetic model reveals no significant change in variance decomposition of SRH from before to during the pandemic, with a heritability estimate of $45 \%$ (CI 36-52\%). For QoL, variance decomposition in Mendel reveals an increase in unique environmental variance during the pandemic ( 0.30 to 1.08$)$, and a decrease in the heritability estimate from $30.9 \%$ to $15.5 \%$. Conclusions: While SRH seems to have been less impacted by the first lockdown, we see immediate changes for QoL. We hypothesize that the increased environmental variance is the result of lockdown measures not impacting everybody equally. Whether these effects persist over longer periods and how they impact health inequalities remain topics for future investigation.

\section{Value of Twin Studies in Causation Assessment}

Shuai Li, Vivienne Esser, Minh Bui and John L. Hopper

Centre for Epidemiology and Biostatistics, Melbourne School of Population and Global Health, The University of Melbourne, Melbourne, VIC, Australia

We developed a method to make Inference about Causation from Examination of FAmiliaL CONfounding (ICE FALCON) using observational data for related individuals, like twins. ICE FALCON shares conceptual similarities with Mendelian Randomization (MR), a method using genetic variants as instrument variables to assess causation. ICE FALCON in effect uses all familial determinants of the exposure, not just those captured by measured genetic variants, as instrumental variable. We applied ICE FALCON to cross-sectional and longitudinal data, and made comparisons with MR. ICE
FALCON analysis of data from 66 monozygotic twin pairs concluded that body mass index (BMI) has a causal effect on DNA methylation, the same conclusions found from MR analyses of more than 4,000 individuals, providing 2.5 times more information per subject than MR. ICE FALCON analysis suggested that the tracking of BMI over time is consistent with a mixture of longitudinal causation and familial confounding. The causes of BMI tracking cannot be assessed by MR because the lack of valid instrumental variables. Our analyses show that ICE FALCON and MR empirically give the same causal conclusion, and ICE FALCON is more powerful and does not require the assumptions of MR. ICE FALCON can be applied to circumstances in which MR cannot be applied; for example, when there is no a priori genetic knowledge or data to create instrumental variables, or when the assumptions underlying MR analysis are suspect. ICE FALCON demonstrates that twin studies have unique value in causation assessment.

\section{Value of Lifespan Approach in Twin and Family Studies of Epigenetics}

Shuai Li

Centre for Epidemiology and Biostatistics, Melbourne School of Population and Global Health, The University of Melbourne, Melbourne, VIC, Australia

Twin and family studies for the genetic and environmental contributions to the variation in epigenetic measures often include participants of limited age ranges, therefore, the findings apply to the investigated age ranges only, but not for the whole lifespan. We collected genome-wide DNA methylation data for $>5,000$ twins aged 0 92 years, developed novel statistical methods and investigated the lifespan genetic and environmental contributions to the variation in several DNA methylation-based measures. Evidence was found that: 1) Genome-wide average DNA methylation is determined in utero; 2) Epigenetic age is mostly caused by environmental factors including those shared to different extents by relatives while living together; 3) Early-life environmental factors strongly influence DNA methylation variation across the lifespan, and their effects are more marked for heritable sites, sites biologically relevant to the regulation of gene expression, and sites/loci associated with various early-life factors (e.g., maternal smoking, puberty onset) and late-life health conditions (e.g., adiposity, cognitive traits, metabolic traits/disorders). Our approach has provided novel insights into the determinants of DNA methylation measures across the lifespan, and implies that early-life environment could impact later-life health through influencing DNA methylation.

\section{Mammographic Analytic Methods in Twin Studies}

John L. Hopper

Melbourne School of Population and Global Health, The University of Melbourne, VIC, Australia

Mammograms contain information that predicts whether a woman will be diagnosed with breast cancer in the interval up until her next regular scan (interval breast cancer), or at a future (screen detected cancer). Conventional mammographic density (MD), the area of white or bright regions, has been extensively studied but the findings are paradoxical. First, MD is negatively correlated with age and body 
mass index, but both are associated with increased breast cancer risk. Second, while high MD can hide existing tumours and predict interval cancers, it is also associated with increased risk of screen-detected cancers even though it would naturally cause a decrease in screendetected cancers. These paradoxes appear to be explained by confounding with other mammogram risk scores (MRS) that predict inherent risk of breast cancer. These include the area of brightest regions on the mammogram, agnostically discovered textural features not related to density, and newer MRS discovered using artificial intelligence. Each MRS appears to bring new risk information and in doing so the association with conventional MD dissipates. Twin and family studies are finding that the MRS are substantially familial with $\mathrm{rMZ} \sim 0.5$, more than twice than $\mathrm{rDZ}$ and rsibs, but the MRS are at most weakly associated with the latest polygenic risk score (PRS) $(r=.04)$. Given the MRS are the strongest known breast cancer risk factors, they might explain more genetic risk than the known PRS. Finding the genetic basis of the MRS could go a long way to explain the missing heritability of breast cancer. 